G. J. McNinch

Nagoya Math. J.

Vol. 190 (2008), 129-181

\title{
THE CENTRALIZER OF A NILPOTENT SECTION
}

\author{
GEORGE J. MCNINCH
}

\section{To Toshiaki Shoji, with respect and admiration, on the occasion of his 60th birthday}

\begin{abstract}
Let $F$ be an algebraically closed field and let $G$ be a semisimple $F$-algebraic group for which the characteristic of $F$ is very good. If $X \in \operatorname{Lie}(G)=\operatorname{Lie}(G)(F)$ is a nilpotent element in the Lie algebra of $G$, and if $C$ is the centralizer in $G$ of $X$, we show that (i) the root datum of a Levi factor of $C$, and (ii) the component group $C / C^{\circ}$ both depend only on the Bala-Carter label of $X$; i.e. both are independent of very good characteristic. The result in case (ii) depends on the known case when $G$ is (simple and) of adjoint type.

The proofs are achieved by studying the centralizer $\mathcal{C}$ of a nilpotent section $X$ in the Lie algebra of a suitable semisimple group scheme over a Noetherian, normal, local ring $\mathcal{A}$. When the centralizer of $X$ is equidimensional on $\operatorname{Spec}(\mathcal{A})$, a crucial result is that locally in the étale topology there is a smooth $\mathcal{A}$-subgroup scheme $L$ of $\mathcal{C}$ such that $L_{t}$ is a Levi factor of $\mathcal{C}_{t}$ for each $t \in \operatorname{Spec}(\mathcal{A})$.
\end{abstract}

\section{$\S 1$. Introduction}

\subsection{The main results}

Let $E$ and $F$ be algebraically closed fields, and let $G_{E}$ and $G_{F}$ be semisimple algebraic groups over $E$ and $F$ respectively. We are going to assume that the root data of these two groups coincide. Further, we suppose that the characteristic of $E$ is 0 , and that the characteristic of $F$ is very good for $G_{F}$ - see Section 3.8.

Using the Bala-Carter Theorem (4.4.1), we may identify the set of nilpotent orbits of $G_{F}$ in $\operatorname{Lie}\left(G_{F}\right)=\mathfrak{g}_{F}$ with the set of nilpotent orbits of $G_{E}$ in $\operatorname{Lie}\left(G_{E}\right)=\mathfrak{g}_{E}$.

Suppose that the orbits of the nilpotent elements $X_{E} \in \mathfrak{g}_{E}$ and $X_{F} \in \mathfrak{g}_{F}$ are the same under the Bala-Carter identification, let $C_{E}$ be the centralizer

Received December 28, 2006.

2000 Mathematics Subject Classification: 20G15.

Research of the author supported in part by the US National Science Foundation through DMS-0437482. 
of $X_{E}$ in $G_{E}$, and let $C_{F}$ be the centralizer of $X_{F}$ in $G_{F}$.

If $H$ is an algebraic group, one says that a closed subgroup $L \subset H$ is a Levi factor if the connected component $L^{o}$ is reductive and if $H$ is isomorphic as an algebraic group to the semidirect product $L \cdot R_{u}(H)$, where we have written $R_{u}(H)$ for the unipotent radical of $H$.

The groups $C_{F}$ and $C_{E}$ have Levi factors $L_{F} \subset C_{F}$ and $L_{E} \subset C_{E}$. Indeed, this is immediate in characteristic 0 , since a result of Mostow shows every linear group to have a Levi factor in that case; in positive characteristic, existence of a Levi factor for $C_{F}$ may be deduced as a consequence of Premet's recent conceptual proof of the Bala-Carter theorem [Pr 03]; cf. (4.1.1) below. The main results of this paper may now be stated:

THEOREM A. The root datum of the reductive group $L_{F}^{o}$ may be identified with that of $L_{E}^{o}$.

Theorem B. The finite groups $C_{E} / C_{E}^{o}$ and $C_{F} / C_{F}^{o}$ are isomorphic.

When $p$ is not a good prime for $G$, the Bala-Carter parametrization of nilpotent orbits does not hold; cf. [Ca 93, §5.11] and [Ja 04, §5.13-5.15] for examples of "extra" nilpotent orbits for these primes. So our statements must at least exclude "bad" characteristics. We have not, however, attempted to prove our results for semisimple groups in all good characteristics. Instead, we have chosen to prove the theorems of this paper under some "standard" assumptions on $G$; in fact, we will prove Theorems A and B for the $T$-standard reductive groups introduced in Section 3.9. A semisimple group is $T$-standard in case the characteristic is very good for $G$, but the group $\mathrm{GL}_{n}$ is always $T$-standard. Thus, our statements apply, for example, to the group $\mathrm{GL}_{n}$ for any $n$, but not to $\mathrm{SL}_{n}$ when $n \equiv 0(\bmod p)$. Note that the centralizer of a regular nilpotent element in $\mathrm{SL}_{n}$ is the direct product of a connected unipotent group with the group $\mu_{n}$ of $n$-th roots of unity; thus when $n \equiv 0(\bmod p)$, the naive statement of Theorem $\mathrm{B}$ would not be correct for $\mathrm{SL}_{n}$.

In the remainder of this introduction, we give an overview of our strategy of proofs of Theorems A and B. We first observe that - as a consequence of the Bala-Carter Theorem; see (4.4.2) - it suffices to prove these Theorems after making a particular choice for the fields $E$ and $F$. For convenience, we will prove the result when $F$ is an algebraic closure of the finite field $\mathbf{F}_{p}$, and $E$ is some suitable algebraically closed field of characteristic 0 . The proofs will be given in Section 5.8 and Section 7.6. We now give some further details about these proofs. 


\subsection{The instability parabolic}

As already mentioned, we rely on the fact that the nilpotent orbits for the group $G_{E}$ and for the group $G_{F}$ are described by the Bala-Carter theorem; cf. Section 4.4 .

Recall that a key idea behind Premet's recent proof [Pr 03] of the BalaCarter theorem was to use a result in geometric invariant theory - due to Kempf and to Rousseau - which attaches a collection of optimal cocharacters of $G$ to an unstable vector in a linear representation of $G$. Let us explain this a bit more. Write $G$ for one of the groups $G_{E}$ or $G_{F}$. An element $X \in \operatorname{Lie}(G)$ is nilpotent if and only if the closure of its adjoint orbit contains 0 ; such vectors are said to be unstable. The Hilbert-Mumford criterion for instability asserts that an unstable vector for $G$ is also unstable for certain one-dimensional sub-tori of $G$. The Hilbert-Mumford criterion has a more precise form due to Kempf and to Rousseau: there is a class of optimal cocharacters of $G$ whose images exhibit such one dimensional sub-tori. One of the nice features of these optimal cocharacters is that they each define the same parabolic subgroup $P_{X}$ of $G$; this parabolic subgroup is known as the instability parabolic determined by $X$. The instability parabolic subgroups determined by nilpotent elements play an important role in this paper.

When $G$ is a reductive group over an arbitrary field $K$ and when $X \in$ $\operatorname{Lie}(G)(K)$ is nilpotent, one knows e.g. by [Mc 04, Prop. 27] that $P_{X}$ is a $K$-parabolic; cf. 4.1 for more on these matters.

\subsection{The component group when $G$ is of adjoint type}

Let again $G$ be one of the groups $G_{E}$ or $G_{F}$, and suppose that $G$ is of adjoint type. For a reductive group in characteristic zero, Alekseevskiu [Al 79] determined the structure of the group of components $C_{G}(X) / C_{G}(X)^{o}$ for each nilpotent $X \in \operatorname{Lie}(G)$. Sommers [So 98] gave later a more conceptual argument for the determination of these groups.

Moreover, given nilpotent elements $X_{E} \in \operatorname{Lie}\left(G_{E}\right)$ and $X_{F} \in \operatorname{Lie}\left(G_{F}\right)$ with the same Bala-Carter label, one knows for semisimple groups of adjoint type that $C_{E} / C_{E}^{o} \simeq C_{F} / C_{F}^{o}$. For a while, this was known only through case-checking - especially, by the work of Mizuno [Miz 80]. More recently, this isomorphism was proved by Premet [Pr 03] and by McNinch-Sommers [MS 03]. Thus, the assertion of Theorem B is known already provided that $G_{E}$ and $G_{F}$ are of adjoint type. 


\subsection{Group schemes}

The proofs of Theorems A and B are achieved by studying reductive group schemes over more general base schemes. Let us give here a brief overview of the argument.

We consider a normal, local, Noetherian integral domain $\mathcal{A}$ with residue field $k$ and field of fractions $K$. Recall that a point $t \in \operatorname{Spec}(\mathcal{A})$ is the same as a prime ideal $\mathfrak{p}_{t} \subset \mathcal{A}$; write $k(t)$ for the field of fractions of $\mathcal{A} / \mathfrak{p}_{t}$. The closed point $s \in \operatorname{Spec}(\mathcal{A})$ is the maximal ideal of $\mathcal{A}$, so that $k(s)=k$ is the residue field. And the generic point $\eta \in \operatorname{Spec}(\mathcal{A})$ is the prime ideal 0 of $\mathcal{A}$, so that $k(\eta)=K$ is the field of fractions. For any $t \in \operatorname{Spec}(\mathcal{A})$, we write $k(\bar{t})$ for a separable closure of the field $k(t)$.

Let $G$ be a semisimple group scheme over $\mathcal{A}$. For $t \in \operatorname{Spec}(\mathcal{A})$, we write $G_{t}$ for the group $G_{/ k(t)}$ obtained by base-change; thus $G_{/ k(t)}$ is a semisimple group over the field $k(t)$. We insist that the characteristic of $k$ is very good for $G_{s}=G_{/ k}$; it is then immediate that the characteristic of $K$ is very good for $G_{\eta}=G_{/ K}$; see Section 3.8.

An $\mathcal{A}$-section of the Lie algebra $\mathfrak{g}=\operatorname{Lie}(G)$ of $G$ - i.e. an element $X \in \mathfrak{g}(\mathcal{A})$ - is nilpotent if its image $X_{K} \in \mathfrak{g}(K)$ is nilpotent. If $X \in \mathfrak{g}(\mathcal{A})$ is a nilpotent section, write $C=C_{G}(X)$ for the centralizer - thus $C$ is an $\mathcal{A}$-subgroup scheme of $G$. Now, $C_{/ K}$ identifies with the centralizer in $G_{/ K}$ of $X_{K}$, and likewise for $C_{/ k}$. If the groups $C_{/ K}$ and $C_{/ k}$ have the same dimension, we say that $X$ is equidimensional; we prove in that case - see Proposition 5.2 - that the group scheme $C$ is smooth over $\mathcal{A}$.

If $P_{0}$ denotes the instability parabolic subgroup of $G_{/ K}$ determined by the nilpotent element $X_{K} \in \mathfrak{g}(K)$, we prove - see Proposition 5.5 - that there is a parabolic $\mathcal{A}$-subgroup scheme $P \subset G$ for which $P_{/ K}=P_{0}$.

This assertion is immediate in case $\mathcal{A}$ is a discrete valuation ring; see (3.10.3). The general case is a consequence of (2.6.3). Note that for general $\mathcal{A}$ as above, the conclusion of Proposition 5.5 actually holds by construction for a collection of equidimensional sections $X \in \mathfrak{g}(\mathcal{A})$ realized as "Richardson sections"; see Theorem 5.4.

The existence of Richardson sections just mentioned also shows that for $s \in \operatorname{Spec}(\mathcal{A})$ and a nilpotent element $Y \in \mathfrak{g}(k(\bar{s}))$, there is an equidimensional nilpotent section $X \in \mathfrak{g}(\mathcal{A})$ such that $Y$ is geometrically conjugate to the value $X(s) \in \mathfrak{g}(k(s))$ of $X$; moreover, the construction of this $X$ makes clear that the Bala-Carter datum of the nilpotent element $X(t) \in \mathfrak{g}(k(\bar{t}))$ is constant for $t \in \operatorname{Spec}(\mathcal{A})$. 
We may now state a key result: locally in the étale topology of $\operatorname{Spec}(\mathcal{A})$, the centralizer $C$ has a Levi factor. This means that after possibly replacing $\mathcal{A}$ by a finite, étale, local extension, we may find a closed, smooth subgroup scheme $L \subset C$ such that $L^{o}$ is reductive and such that $L_{t}$ is a Levi factor of $C_{t}$ for $t \in \operatorname{Spec}(\mathcal{A})$; cf. Theorem 5.7.

The existence of the Levi factor $L$ essentially settles Theorem A. Note that we also prove - cf. Corollary 5.7 - for any equidimensional nilpotent section $X$ that the Bala-Carter datum of $X(t)$ is constant for $t \in \operatorname{Spec}(\mathcal{A})$.

For Theorem $\mathrm{B}$ one considers the sheaf on the étale site of $\mathcal{A}$ determined by the quotient $C / C^{o} \simeq L / L^{o}$. When $G$ is a semisimple group scheme over $\mathcal{A}$ with adjoint root datum, Theorem $\mathrm{B}$ is known for the geometric fibers of $G$; it follows that the sheaf $L / L^{o}$ is represented by a finite étale group scheme over $\mathcal{A}$. To complete the proof of Theorem B, we must argue when $G$ is no longer adjoint that the sheaf $L / L^{o}$ is still represented by a finite étale group scheme; this is carried out in Section 7.

Theorem A was announced by the author in June 2005 in a talk in the conference on Algebraic Groups and Finite Reductive Groups at the Bernoulli Center of the École Polytechnique Fédérale de Lausanne. The author thanks Jens Carsten Jantzen, Michel Raynaud, and Jean-Pierre Serre for useful remarks during the preparation of this manuscript.

\section{$\S 2$. Some recollections}

\subsection{Assumptions and notation}

Let $\mathcal{A}$ be a Noetherian integral domain. We are going to consider schemes over $\mathcal{A}$, and - as e.g. in [Ja 03] - we will interchangeably regard a scheme over $\mathcal{A}$ either as a set-valued functor on all commutative $\mathcal{A}$-algebras [more precisely: all commutative $\mathcal{A}$-algebras in some universe, to avoid wellknown logical pitfalls] or as the ringed topological space which represents this functor.

Given a scheme $X$ of finite type over $\mathcal{A}$ and a point $x \in X$ we write $\mathscr{O}_{x}$ for the local ring of $x$, and we write $k(x)$ for the residue field of $\mathscr{O}_{x}$. When $X=\operatorname{Spec}(\mathcal{A})$, we write $\mathcal{A}_{x}$ for this local ring. We will denote by $k(\bar{x})$ a separably closed field containing $k(x)$; thus $\bar{x}$ is a geometric point of $X$.

If $t$ is a point of $\operatorname{Spec}(\mathcal{A})$, we write $X_{t}$ for the fiber product $X \times \operatorname{Spec}(\mathcal{A})$ Spec $k(t)$; then $X_{t}$ is a scheme of finite type over the field $k(t)$.

Similarly, if $\mathcal{A} \subset \mathcal{B}$ is an extension, we write $X_{/ \mathcal{B}}$ for the fiber product $X \times_{\operatorname{Spec}(\mathcal{A})} \operatorname{Spec} \mathcal{B}$; then $X_{/ \mathcal{B}}$ is a scheme of finite type over $\mathcal{B}$. 


\subsection{Normal local rings and étale extensions}

Assume that the Noetherian integral domain $\mathcal{A}$ is normal and local. If $\mathcal{B}$ is a commutative ring containing $\mathcal{A}$, then $\mathcal{B}$ is said to be a local extension of $\mathcal{A}$ if $\mathcal{B}$ is itself local, and if the maximal ideal of $\mathcal{A}$ is contained in that of $\mathcal{B}$. We have the following:

(2.2.1). [SGA 1, Exp. I, Prop. 10.1] If $\mathcal{B} \supset \mathcal{A}$ is a local étale extension of finite type, then $\mathcal{B}$ is a domain, the field of fractions $L$ of $\mathcal{B}$ is a finite separable extension of the field of fractions $K$ of $\mathcal{A}$, and $\mathcal{B}$ is the integral closure of $\mathcal{A}$ in L. In particular, $\mathcal{B}$ is Noetherian and is finite over $\mathcal{A}$.

In the language of the étale topology (see [Mil 80, II] or Section 6.1 below), we have:

(2.2.2). Any étale neighborhood $X \rightarrow \operatorname{Spec}(\mathcal{A})$ of the closed point of $\operatorname{Spec}(\mathcal{A})$ contains an affine open $\mathcal{A}$-subscheme $\operatorname{Spec}(\mathcal{B}) \subset X$ for some finite, étale, local $\mathcal{A}$-algebra $\mathcal{B}$.

If $X$ is an $\mathcal{A}$-scheme, then we say that a property of $X$ holds locally in the étale topology of $\mathcal{A}$ if the property holds for the $\mathcal{B}$-scheme $X_{/ \mathcal{B}}$ for a suitable finite, étale, local extension $\mathcal{B}$ of $\mathcal{A}$; note that $\mathcal{B}$ is then necessarily a domain.

\subsection{Smoothness of stabilizers}

In this section, $\mathcal{A}$ is a Noetherian integral domain. Let $G$ be a group scheme which is smooth and of finite type over $\mathcal{A}$, and let $Y$ be a scheme which is flat and of finite type over $\mathcal{A}$. Suppose that $G$ acts on $Y$ and that the action is given by a morphism of $\mathcal{A}$-schemes a : $G \times_{\mathcal{A}} Y \rightarrow Y$.

If $\alpha \in Y(\mathcal{A})$ is an $\mathcal{A}$-section, then for each commutative $\mathcal{A}$-algebra $\Lambda$, the section $\alpha$ determines a section $\alpha_{\Lambda} \in Y(\Lambda)$; for $t \in \operatorname{Spec}(\mathcal{A})$ we write $\alpha(t)$ for the image $\alpha_{k(t)}$ in $Y(k(t))$.

Let now $\alpha, \beta \in Y(\mathcal{A})$ be two $\mathcal{A}$-sections of $Y$. The transporter $\operatorname{Trans}_{G}(\alpha, \beta)$ is the subfunctor of $G$ given for each commutative $\mathcal{A}$-algebra $\Lambda$ by

$$
\operatorname{Trans}_{G}(\alpha, \beta)(\Lambda)=\left\{g \in G(\Lambda) \mid g \cdot \alpha_{\Lambda}=\beta_{\Lambda}\right\} .
$$

In particular, the stabilizer $\operatorname{Stab}_{G}(\alpha)=\operatorname{Trans}_{G}(\alpha, \alpha)$ is the subfunctor of $G$ given by

$$
\operatorname{Stab}_{G}(\alpha)(\Lambda)=\left\{g \in G(\Lambda) \mid g \cdot \alpha_{\Lambda}=\alpha_{\Lambda}\right\}
$$


Write $\mu_{\alpha}$ for the orbit mapping

$$
\mu_{\alpha}: G \longrightarrow Y
$$

determined by the section $\alpha \in Y(\mathcal{A})$; for each commutative $\mathcal{A}$-algebra $\Lambda$ we have $\mu_{\alpha}(h)=\mathbf{a}\left(h, \alpha_{\Lambda}\right)$ for each $h \in G(\Lambda)$. We now regard $\alpha, \beta \in Y(\mathcal{A})$ as sections $S \stackrel{\phi_{\alpha}}{\longrightarrow} Y$ and $S \stackrel{\phi_{\beta}}{\longrightarrow} Y$, where $S$ is the spectrum of $\mathcal{A}$. Then we see that the sub-functor $\operatorname{Trans}_{G}(\alpha, \beta)$ may be identified with the fiber product $G \times \times_{Y, \phi_{\beta}} S$ :

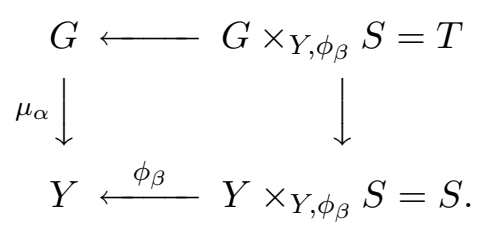

It is thus a subscheme of $G$ which is of finite type over $\mathcal{A}$, and it is closed in $G$ if $\phi_{\beta}$ is a closed embedding. In particular, the $\operatorname{stabilizer~} \operatorname{Stab}_{G}(\alpha)$ is a subscheme of $G$ which is of finite type over $\mathcal{A}$; it is closed in $G$ in case $\phi_{\alpha}$ is a closed embedding.

We are interested in conditions under which the transporter $\operatorname{Trans}_{G}(\alpha, \beta)$ and the stabilizer $\operatorname{Stab}_{G}(\alpha)$ are smooth; we give two such conditions, as follows:

(2.3.1). Suppose for each $s \in \operatorname{Spec}(\mathcal{A})$ that the $G_{s}$-orbit of $\alpha(s)$ in $Y_{s}$ is separable and dense. Then for each section $\beta \in Y(\mathcal{A})$, the transporter $T=$ $\operatorname{Trans}_{G}(\alpha, \beta)$ is a smooth $\mathcal{A}$-subscheme of $G$. In particular, the stabilizer $C=\operatorname{Stab}_{G}(\alpha)$ is a smooth $\mathcal{A}$-subgroup scheme of $G$.

Proof. If $\mu_{\alpha}$ denotes the orbit map as above, we claim first that $\mu_{\alpha}$ : $G \rightarrow Y$ is smooth. Fix a point $g \in G$; we argue that $\mu_{\alpha}$ is smooth at g. Indeed, $G$ is smooth over $\mathcal{A}, Y$ is flat over $\mathcal{A}$, and $\mathbf{a}$ is of finite type. Thus according to [SGA 1, Exp. II, Cor. 2.2], the smoothness of $\mu_{\alpha}$ at $g$ will follow provided that $\mu_{\alpha, s}: G_{s} \rightarrow Y_{s}$ is smooth at $g$, where $s \in \operatorname{Spec}(\mathcal{A})$ is the image of $g$ under the projection $G \rightarrow \operatorname{Spec}(\mathcal{A})$; since the $G_{s}$-orbit of $\alpha(s)$ is separable and dense, $\mu_{\alpha, s}$ is indeed smooth at $g$.

Since smooth morphisms are stable under base-change [SGA 1, Exp. II, Prop. 1.3], and since $\mu_{\alpha}$ is smooth, it follows that $T$ and $C$ are smooth over $\operatorname{Spec}(\mathcal{A})$, as required.

(2.3.2). Assume that the Noetherian integral domain $\mathcal{A}$ is normal. 
(1) If $\operatorname{Trans}_{G}(\alpha, \beta)_{t}$ is smooth over $k(t)$ for each $t \in \operatorname{Spec}(\mathcal{A})$, and if the irreducible components of $\operatorname{Trans}_{G}(\alpha, \beta)_{t}$ all have the same dimension independent of $t \in \operatorname{Spec}(\mathcal{A})$, then $\operatorname{Trans}_{G}(\alpha, \beta)$ is a smooth $\mathcal{A}$ subscheme of $G$.

(2) If $C_{G}(X)_{t}$ is smooth over $k(t)$ for each $t \in \operatorname{Spec}(\mathcal{A})$, and if the irreducible components of $C_{G}(X)_{t}$ all have the same dimension independent of $t \in \operatorname{Spec}(\mathcal{A})$, then $C_{G}(X)$ is a smooth $\mathcal{A}$-subgroup scheme of G.

Proof. Of course, (2) is a special case of (1). Since the scheme $\operatorname{Trans}_{G}(X, Z)$ is of finite type over $\mathcal{A}$ and since $\mathcal{A}$ is normal, (1) follows from [SGA 1, Exp. II, Prop. 2.3].

\subsection{Henselian rings}

Suppose that the Noetherian domain $\mathcal{A}$ is moreover local. Then $\mathcal{A}$ is said to be Henselian if the conclusion of Hensel's lemma holds for $\mathcal{A}$ (see e.g. [Mil 80, I §4]). The local domain $\mathcal{A}$ is said to be strictly Henselian if it is Henselian and if its residue field is separably closed.

If $\mathfrak{m}$ is the maximal ideal of $\mathcal{A}$, recall that $\mathcal{A}$ is Henselian if it is complete in its $\mathfrak{m}$-adic topology [Mil 80, I, Prop. 4.5]. Given any local domain $\mathcal{A}$, we can construct its Henselization $\mathcal{A}^{\mathrm{h}}[\mathrm{Mil} 80, \mathrm{I} \S 4]$ and its strict Henselization $\mathcal{A}^{\text {sh }}$ loc. cit.

Let $X$ be a smooth scheme of finite type over $\mathcal{A}$, and write $k$ for the residue field of $\mathcal{A}$.

(2.4.1). [Mil 80, I Exerc. 4.13] If $\mathcal{A}$ is Henselian, the natural map $X(\mathcal{A}) \rightarrow X(k)$ is surjective.

Suppose that $G$ is a smooth group scheme of finite type over $\mathcal{A}$. Let $Y$ be a scheme which is flat and of finite type over $\mathcal{A}$, and assume that $G$ acts on $Y$ by $\mathcal{A}$-morphisms.

(2.4.2). Let $\alpha, \beta \in Y(\mathcal{A})$, suppose that the $G_{t}$-orbit of $\alpha(t)$ is separable and dense in $Y_{t}$ for every $t \in \operatorname{Spec}(\mathcal{A})$, and suppose that the elements $\alpha(s), \beta(s) \in Y(k(s))=Y(k)$ are conjugate by an element of $G(k)$, where $s$ is the closed point of $\operatorname{Spec}(\mathcal{A})$. Then locally in the étale topology of $\mathcal{A}$, the sections $\alpha$ and $\beta$ are conjugate by a section of $G$. More precisely, there is a finite, étale, local extension $\mathcal{B}$ of $\mathcal{A}$ such that $\alpha$ and $\beta$ are conjugate by an element of $G(\mathcal{B})$. 
Proof. Indeed, by (2.3.1), the transporter $\operatorname{Trans}_{G}(\alpha, \beta)$ is a smooth subscheme of $G$. Thus by $(2.4 .1)$ any $k$-point of the transporter may be lifted to an $\mathcal{A}^{\mathrm{h}}$-point, where $\mathcal{A}^{\mathrm{h}}$ is the Henselization. Now the result follows from the construction of $\mathcal{A}^{\mathrm{h}}$ as the limit of étale neighborhoods of $\mathcal{A}$ [Mil 80, I $§ 4]$.

\subsection{Dimensions of fibers}

Let $X$ be a scheme of finite type over the Noetherian domain $\mathcal{A}$. Let us write $\pi: X \rightarrow S=\operatorname{Spec}(\mathcal{A})$ for the structure morphism. Then Chevalley's upper semicontinuity theorem - cf. [EGA IV, Thm. 13.1.3] - gives rough information on the fibers, as follows:

(2.5.1). For each integer $n$, the set of $x \in X$ such that $\operatorname{dim}_{x} \pi^{-1}(\pi(x))$ $\geq n$ is closed in $X$.

Now suppose that $\mathcal{A}$ is a local, Noetherian domain. Let $\eta \in S$ be the generic point and let $s \in \operatorname{Spec}(\mathcal{A})$ be the closed point.

(2.5.2). If $e=\operatorname{dim} \pi^{-1} \eta=\operatorname{dim} X_{\eta}$ and $f=\operatorname{dim} \pi^{-1} s=\operatorname{dim} X_{s}$, then for each $t \in \operatorname{Spec}(\mathcal{A})$ we have $f \geq \operatorname{dim} X_{t} \geq e$.

Proof. Let $t \in \operatorname{Spec}(\mathcal{A})$. Arguing as in [EGA IV, Cor. 13.1.6], assertion (2.5.1) shows that $\operatorname{dim} X_{t} \geq e$. On the other hand, let $\mathfrak{p} \subset \mathcal{A}$ be the prime ideal corresponding to $t$. We may form the fiber product $X \times{ }_{\operatorname{Spec}(\mathcal{A})}$ $\operatorname{Spec}(\mathcal{A} / \mathfrak{p})$. The morphism $\operatorname{Spec}(\mathcal{A} / \mathfrak{p}) \rightarrow \operatorname{Spec}(\mathcal{A})$ is just the inclusion of the closure of $\{t\}$ in $\operatorname{Spec}(\mathcal{A})$; in particular, $t$ is (identified with) the generic point of $\operatorname{Spec}(\mathcal{A} / \mathfrak{p})$, and $s$ remains the closed point. For any point $r \in \operatorname{Spec}(\mathcal{A} / \mathfrak{p}) \subset \operatorname{Spec}(\mathcal{A})$, the fiber over $r$ of $X \times_{\operatorname{Spec}(\mathcal{A})} \operatorname{Spec}(\mathcal{A} / \mathfrak{p})$ identifies with $X_{r}$. Thus the inequality $f \geq \operatorname{dim} X_{t}$ results from the inequality already established.

\subsection{Existence of sections}

In this section, we consider a Noetherian, normal domain $\mathcal{A}$.

(2.6.1). Let $Y$ be an affine $\mathcal{A}$-scheme of finite type, and let $x_{\eta} \in$ $Y(k(\eta))$, where $\eta$ is the generic point of $\operatorname{Spec}(\mathcal{A})$. For each prime ideal of height one $\mathfrak{p} \subset \mathcal{A}$, suppose that $x_{\mathfrak{p}} \in Y\left(\mathcal{A}_{\mathfrak{p}}\right)$, and that the image of $x_{\mathfrak{p}}$ under the natural map $Y\left(\mathcal{A}_{\mathfrak{p}}\right) \rightarrow Y(k(\eta))$ is $x_{\eta}$. Then there is a section $x \in Y(\mathcal{A})$ such that the image of $x$ under the natural map $Y(\mathcal{A}) \rightarrow Y\left(\mathcal{A}_{\mathfrak{p}}\right)$ is $x_{\mathfrak{p}}$ for each prime ideal $\mathfrak{p}$ of height one. 
Proof. Write $Y=\operatorname{Spec}(\mathcal{B})$ for the $\mathcal{A}$-algebra $\mathcal{B}=\mathcal{A}\left[y_{1}, \ldots, y_{n}\right]$. The point $x_{\eta}$ is the same as an $\mathcal{A}$-homomorphism $f_{\eta}: \mathcal{B} \rightarrow k(\eta)$. For each prime ideal $\mathfrak{p} \subset \mathcal{A}$ of height one, the assumptions mean that $f_{\eta}\left(y_{i}\right) \in \mathcal{A}_{\mathfrak{p}} \subset k(\eta)$ for $1 \leq i \leq n$, and that the resulting homomorphism $f_{\mathfrak{p}}: \mathcal{B} \rightarrow \mathcal{A}_{\mathfrak{p}}$ determines the point $y_{\mathfrak{p}}$.

Since $\mathcal{A}$ is normal, one knows that

$\mathcal{A}=\bigcap_{\mathfrak{p}} \mathcal{A}_{\mathfrak{p}}$, the intersection taken over all prime ideals $\mathfrak{p} \subset \mathcal{A}$ of height 1 ;

see e.g. [Li 02, Lem. 4.1.13]. We conclude that $f_{\eta}\left(y_{i}\right) \in \mathcal{A}$ for $1 \leq i \leq n$; writing $x \in Y(\mathcal{A})$ for the section determined by the resulting homomorphism $f: \mathcal{B} \rightarrow \mathcal{A}$, the result follows.

Fix a projective $\mathcal{A}$-scheme $X$ of finite type. Recall now the following (see for instance [Li 02, Theorem 3.3.25]):

(2.6.2). If $\mathcal{A}$ is a discrete valuation ring, then the natural mapping $X(\mathcal{A}) \rightarrow X(k(\eta))$ is bijective, where $\eta$ is the generic point of $\operatorname{Spec}(\mathcal{A})$.

Suppose given an element $x_{F} \in X(F)$ for each $\mathcal{A}$-algebra $F$ that is a field. Whenever $\mathcal{B}$ is an $\mathcal{A}$-algebra that is a discrete valuation ring with field of fractions $F$, write $x_{\mathcal{B}} \in X(\mathcal{B})$ for the section determined by $X(F)$. We make two assumptions:

(S1) Whenever the $\mathcal{A}$-algebras $F_{1}, F_{2}$ are fields satisfying $F_{1} \subset F_{2}$, suppose that $x_{F_{2}}$ coincides with the image of $x_{F_{1}}$ under the natural map $X\left(F_{1}\right) \rightarrow X\left(F_{2}\right)$.

(S2) Whenever $\mathcal{B}$ is an $\mathcal{A}$-algebra that is a discrete valuation ring with field of fractions $F$ and residue field $\mathfrak{f}$, we suppose that $x_{\mathfrak{f}}$ coincides with the image of $x_{\mathcal{B}}$ under the natural map $X(\mathcal{B}) \rightarrow X(\mathfrak{f})$.

(2.6.3). Under the hypotheses (S1) and (S2), there is a unique section $x \in X(\mathcal{A})$ such that for each $\mathcal{A}$-algebra $F$ that is a field, the element $x_{F}$ is the image of $x$ under the natural map $X(\mathcal{A}) \rightarrow X(F)$.

Proof. First note that uniqueness of the section $x$ is immediate, e.g. since the image of $x$ in $X(k(\eta))$ must coincide with $x_{k(\eta)}$.

We now prove the existence of $x$. In view of the uniqueness of the section $x$, it suffices to construct $x$ locally on $\operatorname{Spec}(A)$; thus, we may and 
will suppose that $\mathcal{A}$ is moreover local. Write $s \in \operatorname{Spec}(\mathcal{A})$ for the unique closed point, and write $k=k(s)$.

Before beginning the proof, choose a very ample invertible sheaf $\mathcal{L}$ on $X$; thus $\mathcal{L}=i^{*} \mathcal{O}(1)$ for a suitable closed embedding $i: X \rightarrow \mathbf{P}_{/ \mathcal{A}}^{n}$. Let $t_{0}, t_{1}, \ldots, t_{n} \in \mathcal{L}(X)$ be global sections such that for each $0 \leq i \leq n$, the set $X_{t_{i}}$ is an affine open $\mathcal{A}$-subscheme, and the affine opens $X_{t_{i}}$ cover $X$.

The proof proceeds by induction on $d=\operatorname{dim} \mathcal{A}$.

When $d=1$, the domain $\mathcal{A}$ is itself a discrete valuation ring and the existence of the desired section $x \in X(\mathcal{A})$ follows immediately from (2.6.2).

Suppose now that $d>1$ and suppose that the result is true in dimension strictly less than $d$. Let $\mathfrak{p}$ denote a height one prime ideal of $\mathcal{A}$. Then the quotient $\mathcal{A} / \mathfrak{p}$ is a Noetherian, normal, local domain of dimension $d-1$. For each $\mathcal{A} / \mathfrak{p}$-algebra $F$ which is a field, we have of course the section $x_{F}$, and it is clear that these sections satisfy conditions (S1) and (S2) for the $\operatorname{ring} \mathcal{A} / \mathfrak{p}$. Thus, the induction hypothesis gives now a section $x^{\prime} \in X(\mathcal{A} / \mathfrak{p})$ whose image in $X(F)$ coincides with $x_{F}$ for each $\mathcal{A} / \mathfrak{p}$-algebra $F$ that is a field.

Since $\mathfrak{p}$ has height one and since $\mathcal{A}$ is normal, the localization $\mathcal{A}_{\mathfrak{p}}$ is a discrete valuation ring. The residue field of $\mathcal{A}_{\mathfrak{p}}$ is $k(\mathfrak{p})$, the field of fractions of $\mathcal{A} / \mathfrak{p}$. By the result when $d=1$, we may find a section $x_{\mathfrak{p}} \in X\left(\mathcal{A}_{\mathfrak{p}}\right)$ whose image in $X(k(\mathfrak{p}))$ coincides with the image of $x^{\prime}$.

Since $X_{t_{j}}$ is an affine $\mathcal{A}$-scheme for each $j$, we may now apply (2.6.1) to the restriction to $X_{t_{j}}$ of the sections $\left\{x_{\mathfrak{p}}\right\}$; we then find the required section $x \in X(\mathcal{A})$.

\section{§3. Reductive groups}

We are going to work throughout the remainder of the paper with a local, normal, Noetherian domain $\mathcal{A}$. Write $K$ for the field of fractions of $\mathcal{A}$ and $k$ for its residue field. Also, write $\eta \in \operatorname{Spec}(\mathcal{A})$ for the generic point; thus $K=k(\eta)$.

\subsection{Group schemes of multiplicative type}

An $\mathcal{A}$-group scheme $D$ is said to be diagonalizable if there is a finitely generated Abelian group such that $D \simeq D_{\Gamma}$, where $D_{\Gamma}=\operatorname{Spec} \mathcal{A}[\Gamma]$; here $\mathcal{A}[\Gamma]$ is the group algebra of $\Gamma$ - i.e. the algebra of those $\mathcal{A}$-valued functions on $\Gamma$ having finite support - made into a Hopf algebra as usual. An $\mathcal{A}$-group scheme $M$ is of multiplicative type if it is diagonalizable locally in the étale topology of $\mathcal{A}$; this means that there is a finite, étale extension $\mathcal{B} \supset \mathcal{A}$ such that $M_{/ \mathcal{B}}$ is diagonalizable. 
An $\mathcal{A}$-group scheme $T$ is a torus if it is a group of multiplicative type and if locally in the étale topology of $\mathcal{A}$ the group $T$ is of the form $D_{\Gamma}$ where $\Gamma$ is finitely generated and free. The torus $T$ is split over $\mathcal{A}$ if it is isomorphic to $D_{\Gamma}$ as an $\mathcal{A}$-group scheme.

\subsection{Reductive group schemes}

Recall that a group scheme $G$ over $\mathcal{A}$ is said to be reductive provided that $G$ is smooth and of finite type over $\mathcal{A}$ and that the fiber $G_{\bar{t}}$ is a (connected and) reductive algebraic group for each algebraically closed geometric point $\bar{t}$ of $\operatorname{Spec}(\mathcal{A})$. The reductive $G$ is moreover semisimple if all $G_{\bar{t}}$ are semisimple algebraic groups.

If $G$ is a group scheme and $T \subset G$ is a subgroup scheme, one says that $T$ is a maximal torus if it is a torus, and if $T_{t}$ is a maximal torus in $G_{t}$ for each point $t$ of $\operatorname{Spec}(\mathcal{A})$. A result of Grothendieck says:

(3.2.1). [SGA 3, Exp. XIV, Cor. 3.20] Any reductive group has a maximal torus.

The reductive group $G$ is said to be split if it has a split maximal torus $T$. If $G$ has a split maximal torus $T$, the root datum of $G$ with respect to $T$ is $\mathcal{R}=\left(X, Y, R, R^{\vee}\right)$ where $X=X^{*}(T)$ is the character group of $T$, $Y=X_{*}(T)$ is the group of cocharacters of $T, R \subset X$ is the set of roots, and $R^{\vee}$ is the set of coroots. We have the following existence theorem of Chevalley:

(3.2.2). [SGA 3, Exp. XXV, Cor. 1.2] Let $\mathcal{R}$ be a root datum. Then there is a split reductive group scheme over $\mathcal{A}$ with the root datum $\mathcal{R}$.

A root datum $\mathcal{R}=\left(X, Y, R, R^{\vee}\right)$ is said to be of adjoint type if $X=\mathbf{Z} R$. Given any root datum, one constructs the corresponding adjoint root datum $\mathcal{R}_{\text {ad }}$ and the morphism $h: \mathcal{R}_{\text {ad }} \rightarrow \mathcal{R}$ of root data, as in [SGA 3, Exp. XXI, Prop. 6.5.5]. Let $G_{\text {ad }}$ be a split semisimple $\mathcal{A}$-group scheme with split maximal torus $T^{\prime}$ and root datum $\mathcal{R}_{\text {ad }}$.

(3.2.3). [SGA 3, Exp. XXIII, Thm. 4.1] There is a unique morphism of $\mathcal{A}$-group schemes $f: G \rightarrow G_{\mathrm{ad}}$ which defines upon restriction to $T$ a morphism $f_{\mid T}: T \rightarrow T^{\prime}$ and induces the mapping $h$ on root data. 


\subsection{Levi factors}

Let $H$ be a smooth and separated ${ }^{1}$ group scheme of finite type over $\mathcal{A}$.

Suppose that there exists a closed subgroup scheme $L \subset H$ such that $L$ is smooth over $\mathcal{A}$ and $L^{o}$ is reductive. Then we say that $L$ is a Levi factor of $H$ if for each $t \in \operatorname{Spec}(\mathcal{A})$ the inclusion $L_{t} \subset H_{t}$ induces an isomorphism of $k(\bar{t})$-group schemes $L_{\bar{t}} \simeq H_{\bar{t}} / R_{u}\left(H_{\bar{t}}\right)$, where $R_{u}\left(H_{\bar{t}}\right)$ is the unipotent of $H_{\bar{t}}$ and $\bar{t}$ is a geometric point over $t$. Equivalently put: for each $t \in \operatorname{Spec}(\mathcal{A})$, the subgroup $L_{t}$ is a Levi factor of $H_{t}$ in the usual sense of linear algebraic groups.

(3.3.1). Let $L \subset H$ be a Levi factor. Write $i: L \rightarrow H$ for the inclusion, and suppose that there is a homomorphism $\rho: H \rightarrow L$ of group schemes over $\mathcal{A}$ such that $\rho \circ i=\mathrm{id}_{L}$; in other words, $\rho$ is a "retraction". Let $R=\operatorname{ker} \rho$. If $\rho$ is smooth, then the mapping $\Phi: L \ltimes R \rightarrow H$ induced by the natural inclusions $L \rightarrow H$ and $R \rightarrow H$ is an isomorphism, where $L \ltimes R$ is the semidirect product group scheme.

Proof. The kernel $R=\rho^{-1}(1)$ identifies with the fiber product $H \times_{L, \rho}$ $\operatorname{Spec}(\mathcal{A})$; since $\rho$ is assumed to be smooth, $R$ is smooth over $\mathcal{A}$.

Since $L_{t}$ is a Levi factor of $H_{t}$ for each $t, \Phi_{t}$ is an isomorphism for each $t \in \operatorname{Spec}(\mathcal{A})$. We have seen that $R$ is smooth, so the group $L \ltimes R$ is smooth. Since both $L \ltimes R$ and $H$ are smooth - hence flat - over $\mathcal{A}$, it now follows from [SGA 1, Exp. I, Prop. 5.7] that $\Phi$ is itself an isomorphism.

\subsection{The identity component}

Let again $H$ be a smooth and separated group scheme of finite type over $\mathcal{A}$.

(3.4.1). There is a smooth, normal, and open subgroup scheme $H^{\circ} \subset$ $H$ which is the union of the connected components of the groups $H_{t}$ for $t \in \operatorname{Spec}(\mathcal{A})$.

For us, an important property of the connected component is the following:

(3.4.2). If $H^{o}$ is reductive, then $H^{o}$ is closed in $H$.

\footnotetext{
${ }^{1}$ Note that we will consider only group schemes $H$ which are affine over $\mathcal{A}$, and thus automatically separated.
} 
Proof. Since $H^{o}$ is reductive, there is a maximal torus $T \subset H^{o}$. Now, it follows from [SGA 3, Exp. XIX, Thm. 2.5] that the Weyl group $W=N_{H^{o}}(T) / C_{H^{o}}(T)=N_{H^{o}}(T) / T$ is (represented by) a finite étale group scheme over $\mathcal{A}$. Since $H$ is assumed to be a separated group scheme over $\mathcal{A}$, it follows from [SGA 3, Exp. XVI, Cor. 1.4] that the inclusion $H^{o} \subset H$ is a closed immersion, as required.

\subsection{Central isogenies}

Let $G$ and $G^{\prime}$ be reductive groups over $\mathcal{A}$. An $\mathcal{A}$-homomorphism $f$ : $G \rightarrow G^{\prime}$ is said to be a central isogeny if $f$ is faithfully flat, finite and if ker $f$ is a central subgroup of $G$. If $f$ is an étale central isogeny, observe that $f_{s}$ and $f_{\eta}$ are separable central isogenies in the usual sense of an algebraic group over a field.

(3.5.1). Let $f: G \rightarrow G^{\prime}$ be an étale central isogeny. Then ker $f$ is a closed and central subgroup scheme of $G$ which is finite, étale and of multiplicative type over $\mathcal{A}$.

Proof. Let $D=\operatorname{ker} f$. Since $f: G \rightarrow G^{\prime}$ is finite and étale, upon base change we see that $D=\operatorname{ker} f \rightarrow \operatorname{Spec}(\mathcal{A})$ is finite and étale as well. To see that $D$ is of multiplicative type, we may replace $\mathcal{A}$ by a finite, étale, local extension and show that $D$ is diagonalizable. Thus, using [SGA 3, Exp. XXII, Cor. 4.2.13] we may suppose that there are split maximal tori $T \subset G$ and $T^{\prime} \subset G^{\prime}$ such that $f_{\mid T}$ factors as a morphism $f_{\mid T}: T \rightarrow T^{\prime}$. Since ker $f$ is étale over $\mathcal{A}$, it follows from [SGA 3, Exp. XXII, Cor. 4.2.8] that ker $f=\operatorname{ker} f_{\mid T}$, so that ker $f$ is a closed subgroup of the torus $T$. It now follows from [SGA 3, Exp. VII, Cor. 3.4] that ker $f$ is diagonalizable, as required.

\subsection{Some centralizers}

Let $H$ be a group scheme which is smooth of finite type over $\mathcal{A}$. Let $D \subset H$ be a subgroup scheme of multiplicative type.

(3.6.1). [SGA 3, Exp. XI, Cor. 5.3] The centralizer $C_{H}(D)$ and the normalizer $N_{H}(D)$ are closed subgroup schemes which are smooth over $\mathcal{A}$.

Now suppose that $G$ is a reductive $\mathcal{A}$-group scheme and that $D \subset H$ is a smooth subgroup scheme of multiplicative type. 
(3.6.2). The centralizer $C_{G}(D)$ is a closed and smooth subgroup scheme whose identity component $C_{G}(D)^{o}$ is a reductive $\mathcal{A}$-group scheme.

Sketch. Since $C_{G}(D)$ is closed and smooth over $\mathcal{A}$ by (3.6.1), it suffices to show that $C_{G}(D)_{\bar{t}}$ has reductive identity component for each $t \in \operatorname{Spec}(\mathcal{A})$; thus, it is enough to prove the result when $G$ is reductive over an algebraically closed field. In that case, $D$ is diagonalizable, and arguing by induction on $\operatorname{dim} G$ one quickly reduces to two cases: $D$ a torus, in which case $C_{G}(D)$ is a Levi factor of a parabolic subgroup of $G$, and $D$ a cyclic group of order invertible in the field, in which case the result follows from [St 68, Cor. 9.3].

Remark. In fact, the preceding result remains valid for any diagonalizable group $D$. It seems to be difficult to find a reference for this more general fact. In case $D$ is smooth, the assertion that $C_{G}(D)^{o}$ is reductive may also be deduced from a result of Richardson [Ri 82, Prop. 10.1.5]; I thank Gerhard Röhrle for pointing out this reference to me.

(3.6.3). There is a maximal torus of $G$ centralized by D. Moreover, if $T$ is any maximal torus of $G$ which is centralized by $D$, then $D \subset T$.

Proof. Let $M=C_{G}(D)$ be the centralizer of $D$ in $M$. Then by (3.6.2) the identity component $M^{o}$ is a reductive subgroup scheme, hence $M^{o}$ contains a maximal torus $T$ (3.2.1). It follows from [SGA 3, Exp. XII, Prop. 1.17] that $T$ is a maximal torus in $G$ as well.

Since $D$ is central in $M$, [SGA 3, Exp. XII, Lem. 4.5] shows for any maximal torus $T$ of $M^{o}$ that the inclusion $D \subset M$ factors through $T$; i.e. $D$ is a subgroup scheme of $T$ as required.

(3.6.4). Let $T \subset G$ be a torus. Then $C_{G}(T)$ is a closed and reductive subgroup scheme.

Proof. In view of (3.6.2), it only remains to show that $C_{G}(T)=$ $C_{G}(T)^{o}$, i.e. that $C_{G}(T)_{x}$ is connected for every $x \in \operatorname{Spec}(\mathcal{A})$; that requirement follows e.g. from [SGA 3, Exp. XIX, §1.3] or from [Sp 98, Theorem 6.4.7]. 


\subsection{The derived group}

Let $\operatorname{Der}(G)$ be the derived subgroup scheme of $G$. Then:

(3.7.1). [SGA 3, Exp. XXII, Thm. 6.2.1] $\operatorname{Der}(G)$ is a closed, normal subgroup scheme which is smooth and semisimple over $\mathcal{A}$.

(3.7.2). [SGA 3, Exp. XXII, Prop. 6.2.7] If $T$ is a split maximal torus of $G$, there is a maximal torus $T^{\prime} \subset \operatorname{Der}(G)$ contained in $T$.

\subsection{Good and very good primes}

Let $\mathfrak{f}$ denote an arbitrary field, and let $H$ be a geometrically quasisimple algebraic group over $\mathfrak{f}$ with absolute root system ${ }^{2} R$. The characteristic $p$ of $\mathfrak{f}$ is said to be a bad prime for $R$ in the following circumstances: $p=2$ is bad whenever $R \neq A_{r}, p=3$ is bad if $R=G_{2}, F_{4}, E_{r}$, and $p=5$ is bad if $R=E_{8}$. Otherwise, $p$ is good. [Here is a more intrinsic definition of good prime: $p$ is good just in case it divides no coefficient of the highest root in $R]$.

If $p$ is good, then $p$ is said to be very good provided that either $R$ is not of type $A_{r}$, or that $R=A_{r}$ and $r \not \equiv-1(\bmod p)$.

If $H$ is reductive, one may apply [KMRT, Theorems 26.7 and 26.8] ${ }^{3}$ to see that there is a possibly inseparable isogeny

$$
T \times \prod_{i=1}^{r} H_{i} \longrightarrow H
$$

for some f-torus $T$ and some $r \geq 1$, where for $1 \leq i \leq r$ there is an isomorphism $H_{i} \simeq R_{E_{i} / \mathfrak{f}} J_{i}$ for a finite separable field extension $E_{i} / \mathfrak{f}$ and a geometrically quasisimple, simply connected $E_{i}$-group scheme $J_{i}$; here, $R_{E_{i} / \mathfrak{f}} J_{i}$ denotes the "Weil restriction" of $J_{i}$ to $\mathfrak{f}$.

Then $p$ is good, respectively very good, for $H$ if and only if that is so for $J_{i}$ for every $1 \leq i \leq r$. Let $\mathfrak{f}_{\text {alg }}$ be an algebraic closure of $F$. Since the groups $J_{i / \mathfrak{f}_{\text {alg }}}$ are uniquely determined by $H_{/ \mathfrak{f}_{\text {alg }}}$ up to central isogeny, the notions of good and very good primes depend only on the group $H_{/ f_{\text {alg }}}$, and these notions depend only on the central isogeny class of the derived group of $H_{/ \mathfrak{f}_{\text {alg }}}$.

\footnotetext{
${ }^{2}$ The absolute root system of $G$ is the root system of $G_{/ \mathfrak{f} \text { sep }}$ where $\mathfrak{f}_{\text {sep }}$ is a separable closure of $\mathfrak{f}$.

${ }^{3}[\mathrm{KMRT}]$ only deals with the semisimple case; the extension to a general reductive group is not difficult to handle, and an argument is sketched in the footnote found in [MT 07, §2.4].
} 
Let now $G$ be a split semisimple scheme over $\mathcal{A}$ with split maximal torus $T$ and corresponding root datum $\mathcal{R}$. Suppose that the characteristic of the residue field $k$ is very good for $G_{s}=G_{/ k}$. For any point $t$ of $\operatorname{Spec}(\mathcal{A})$, either the characteristic of $k(t)$ is zero, or is the same as the characteristic of $k$; thus also the characteristic of $k(t)$ is very good for $G_{t}$.

(3.8.1). Let $f: G \rightarrow G_{\mathrm{ad}}$ be the morphism of (3.2.3), where $G_{\mathrm{ad}}$ is the $\mathcal{A}$-group scheme with the root datum $\mathcal{R}_{\mathrm{ad}}$. Then $f$ is an étale central isogeny.

Proof. That $f$ is a central isogeny follows from [SGA 3, Exp. XXII, Prop. 4.2.10]. To see that $f$ is étale, it suffices by [SGA 1, Exp. I, Cor. 5.9] to observe that the mapping $f_{t}: G_{t} \rightarrow G_{\mathrm{ad}, t}$ is étale for each $t$ in $\operatorname{Spec}(\mathcal{A})$. In view of our assumptions on the characteristic, one may use the descriptions found in $[\mathrm{Hu} 95,0.13]$ to see that the tangent mapping $d f_{t}$ must be an isomorphism, whence the required assertions.

\subsection{Strongly standard reductive group}

Consider reductive groups $H$ over $\mathcal{A}$ which are direct products

$$
\text { (*) } \quad H=H_{1} \times_{S} T
$$

where $T$ is a torus over $\mathcal{A}$, and where $H_{1}$ is a semisimple group over $\mathcal{A}$ such that the characteristic of $K$ is very good for $H_{1 / K}$ and such that the characteristic of $k$ is very good for $H_{1 / k}$.

Let $G$ be a reductive $\mathcal{A}$-group. Then $G$ will be said to be $D$-standard if there exists a reductive group $H$ of the form $(*)$, an $\mathcal{A}$-subgroup $D \subset H$ of multiplicative type, and an étale $\mathcal{A}$-isogeny between $G$ and the $\mathcal{A}$-group $C_{H}(D)^{o} 4$.

Similarly, $G$ will be said to be $T$-standard if there exists a reductive group $H$ of the form (*), an $\mathcal{A}$-torus $T \subset H$, and an étale $\mathcal{A}$-isogeny between $G$ and the smooth reductive $\mathcal{A}$-group $C_{H}(T)$.

Of course, any $T$-standard group is $D$-standard.

Remark. Let $\mathfrak{f}$ be a field. In [Mc 05], the term strongly standard reductive group was used for what we call here a $T$-standard group scheme over $\mathfrak{f}$. In [MT 07], the term strongly standard reductive group was used for what we call here a $D$-standard group scheme over $\mathfrak{f}$.

\footnotetext{
${ }^{4}$ If there is such an isogeny, then $C_{H}(D)^{o}$ is of course reductive, so the definition is independent of (3.6.2); in particular, there is no need to insist in the definition that $D$ be smooth.
} 
We have evidently

(3.9.1). If $G$ is D-standard, respectively $T$-standard, then for $t \in$ $\operatorname{Spec}(\mathcal{A})$, the fiber $G_{t}$ is D-standard, respectively $T$-standard.

(3.9.2). Let $G$ be a D-standard reductive group over a field $\mathfrak{f}$, let $g \in$ $G(\mathfrak{f})$ and $X \in \mathfrak{g}(\mathfrak{f})$. Then the centralizers $C_{G}(g)$ and $C_{G}(X)$ are smooth $\mathfrak{f}$-subgroup schemes of $G$.

Proof. [MT 07, Prop. 12].

(3.9.3). Let $\mathcal{L}$ be a free $\mathcal{A}$-module of finite rank $n$. Then the reductive $\mathcal{A}$-group $\mathrm{GL}(\mathcal{L})$ is $T$-standard (hence also D-standard).

Sketch. Indeed, let $p$ denote the characteristic of the residue field $k$. If $p=0$, nothing needs to be said, so assume $p>0$. If $n \not \equiv 0(\bmod p)$ then $H=\operatorname{SL}(\mathcal{L}) \times \mathbf{G}_{m}$ is of the form $(*)$, and multiplication defines an étale isogeny $H \rightarrow \mathrm{GL}(\mathcal{L})$.

If $n \equiv 0(\bmod p)$, then $H=\operatorname{SL}(\mathcal{L} \oplus \mathcal{A})$ has the form $(*)$, and $\operatorname{GL}(\mathcal{L})$ is isomorphic to the centralizer in $H$ of a suitable split $\mathcal{A}$-torus $S \subset H$.

\subsection{Parabolic subgroups}

Let $G$ be a reductive group scheme over $\mathcal{A}$, and let $P \subset G$ be an $\mathcal{A}$ subgroup scheme. One says that $P$ is an $\mathcal{A}$-parabolic subgroup scheme of $G$ if $P$ is smooth over $\mathcal{A}$ and if $P_{t}$ is a parabolic subgroup of $G_{t}$ for each point $t$ of $\operatorname{Spec}(\mathcal{A})$.

We recall the following:

(3.10.1). [SGA 3, Exp. XXVI, Cor. 3.5] Consider the functor Par defined for commutative $\mathcal{A}$-algebras $\Lambda$ by the rule

$$
\underline{\operatorname{Par}}(\Lambda)=\text { set of all } \Lambda \text {-parabolic subgroup schemes of } G_{/ \Lambda} \text {. }
$$

Then $\underline{\mathrm{Par}}$ is (represented by) a smooth and projective scheme over $\operatorname{Spec}(\mathcal{A})$.

(3.10.2). Let $P, Q \subset G$ be $\mathcal{A}$-parabolic subgroup schemes, and write $\eta$ for the generic point of $\operatorname{Spec}(\mathcal{A})$.

(a) If $P_{\eta}=Q_{\eta}$, then $P=Q$.

(b) If $P_{\eta}$ and $Q_{\eta}$ are conjugate by an element of $G(k(\eta))$, then $P=$ $\operatorname{Int}(g) Q$ for a section $g \in G(\mathcal{A})$. 
Proof. For (a), note first that by (3.10.1) the scheme $\underline{\text { Par }}$ of parabolic subgroups of $G$ is projective - hence in particular, separated - over $\mathcal{A}$. Thus if the restrictions of two $\mathcal{A}$-sections of Par to the dense open subset $\{\eta\} \subset \operatorname{Spec}(\mathcal{A})$ coincide, then the sections coincide by [Li 02, Prop. 3.3.11].

For (b), let $\beta(\operatorname{Dyn}(G))$ be the scheme of types of the parabolic subgroup schemes of $G$, and for an $\mathcal{A}$-parabolic subgroup scheme $P$ of $G$, we will write $\underline{t}(P) \in \beta(\operatorname{Dyn}(G))(\mathcal{A})$ for the type of $P$; cf. [SGA 3, Exp. XXVI, Defn 3.4]. Thus $\underline{t}: \underline{\operatorname{Par}} \rightarrow \beta(\operatorname{Dyn}(G))$ is a morphism of schemes. Since $\{\eta\}$ is dense in $\operatorname{Spec}(\mathcal{A})$ and since $P_{\eta}$ and $Q_{\eta}$ are $G(k(\eta))$-conjugate, it follows that $\underline{t}(Q)=\underline{t}(P)$. Since $\mathcal{A}$ is local, assertion (b) now follows from [SGA 3, Exp. XXVI, Cor. 5.5].

(3.10.3). Assume that $\mathcal{A}$ has dimension 1 ; i.e. assume that $\mathcal{A}$ is a discrete valuation ring. If $Q \subset G_{\eta}=G_{/ K}$ is a $K$-parabolic subgroup, there is a unique parabolic $\mathcal{A}$-subgroup scheme $P \subset G$ inducing $Q$ on base-changei.e. $Q=P_{\eta}$.

Proof. Indeed, uniqueness follows from (3.10.2)(a). Since the scheme $\underline{\text { Par }}$ of parabolic subgroups of $G$ is projective (3.10.1), and since $\mathcal{A}$ is a discrete valuation ring, it follows that its $K$-points are the same as its $\mathcal{A}$ points [Li 02, Theorem 3.3.25].

The validity of (3.10.3) indeed requires some hypothesis on $\mathcal{A}$. Notice that if $G=\mathrm{GL}_{2}$, then the scheme of Borel subgroups of $G$ identifies with the projective line $\mathbf{P}_{\mathcal{A}}^{1}$. If $k$ is a field and $\mathcal{A}$ is the 2 dimensional (regular, hence normal) local domain $\mathcal{A}=k[x, y]_{(x, y)}$, then the $K$-point $(x: y) \in \mathbf{P}_{\mathcal{A}}^{1}(K)$ does not determine an $\mathcal{A}$-section of $\mathbf{P}_{\mathcal{A}}^{1}$.

\subsection{Cocharacters and parabolic subgroups}

If $H$ is an algebraic group over a field $\mathfrak{f}$, a cocharacter of $H$ is an $\mathfrak{f}$-homomorphism $\mathbf{G}_{m} \rightarrow H$. In this paper, we will be interested more generally in homomorphisms of group schemes from the multiplicative group to a given group.

Let $H$ be an $\mathcal{A}$-group scheme, consider a representation of $H$ on a free $\mathcal{A}$-module of finite rank $V$ given by the comodule map $\rho: V \rightarrow V \otimes_{\mathcal{A}} \mathcal{A}[H]$. If $\phi: \mathbf{G}_{m} \rightarrow H$ is an $\mathcal{A}$-homomorphism of group schemes, one obtains a representation of $\mathbf{G}_{m}$ on $V$ with co-module map $\left(1 \otimes \phi^{*}\right) \circ \rho: V \rightarrow$ $V \otimes_{\mathcal{A}} \mathcal{A}\left[\mathbf{G}_{m}\right]=V \otimes_{\mathcal{A}} \mathcal{A}\left[t, t^{-1}\right]$. One now finds a direct sum decomposition $V=\bigoplus_{n \in \mathbf{Z}} V(\phi ; n)$ where $V(\phi ; n)$ is the $n$-weight space; i.e.

$$
V(\phi ; n)=\left\{v \in V \mid\left(1 \otimes \phi^{*}\right) \circ \rho(v)=v \otimes t^{n}\right\} .
$$


We apply this especially when $H$ is smooth and of finite type over $\mathcal{A}$, so that $\mathfrak{h}=\operatorname{Lie}(H)$ is a free $\mathcal{A}$-module of finite rank on which $H$ acts by the adjoint representation.

As to the existence of $\mathcal{A}$-homomorphisms $\mathbf{G}_{m} \rightarrow H$, we first note that since the domain $\mathcal{A}$ is assumed to be normal, we have:

(3.11.1). [SGA 3, Exp. X, Lem. 8.4] Let D, H be group schemes over $\mathcal{A}$, where $D$ is of multiplicative type, and $H$ is smooth. Let $\phi: H_{\eta} \rightarrow D_{\eta}$ be a homomorphism of group schemes over $K=k(\eta)$. Then there is a unique homomorphism $\psi: H \rightarrow D$ of group schemes over $\mathcal{A}$ such that $\phi=\psi_{\eta}$.

An immediate consequence is the following:

(3.11.2). Let $T$ be an $\mathcal{A}$-torus, and let $\phi: \mathbf{G}_{m, \eta} \rightarrow T_{\eta}$ be a cocharacter over $k(\eta)=K$. Then there is a unique $\mathcal{A}$-homomorphism of group schemes $\psi: \mathbf{G}_{m} \rightarrow T$ such that $\phi=\psi_{\eta}$.

Let again $G$ be a reductive group scheme over $\mathcal{A}$. Suppose now that $\phi: \mathbf{G}_{m} \rightarrow G$ is an $\mathcal{A}$-homomorphism of group schemes. Composing $\phi$ with the (left) regular representation of $G$, the algebra $\mathcal{A}[G]$ becomes a locally finite representation of $\mathbf{G}_{m}$; let us write it as the direct sum of its weight spaces

$$
\bigoplus_{n \in \mathbf{Z}} \mathcal{A}[G]_{n} .
$$

Form the ideal $I$ generated by $\sum_{n>0} \mathcal{A}[G]_{n}$. Then $P(\phi)=P_{G}(\phi)=$ $\operatorname{Spec}(\mathcal{A}[G] / I)$ is a closed subgroup scheme of $G$.

(3.11.3). $P(\phi)=P_{G}(\phi)$ is a parabolic subgroup scheme of $G$ with Lie $P(\phi)=\bigoplus_{n \geq 0} \mathfrak{g}(\phi ; n)$.

Proof. It follows from [Sp 98, Prop. 8.4.5 and Theorem 13.4.2] that $P=P(\phi)$ determines a parabolic subgroup of each fiber upon base-change; in particular, $P_{t}$ is smooth over $k(t)$ for each $t \in \operatorname{Spec}(\mathcal{A})$. To see that $P$ is smooth over $\mathcal{A}$, we may first replace $\mathcal{A}$ by an étale local extension and thus suppose the image of $\phi$ to lie in a split maximal torus of $G$. Then $P$ is a standard parabolic and hence smooth.

Let $P$ be any parabolic subgroup scheme of $G$. 
(3.11.4). [SGA 3, Exp. XXVI, Prop. 1.6] There is a largest normal subgroup scheme $R=R_{u}(P) \subset P$ which is smooth over $\mathcal{A}$ and has connected and unipotent geometric fibers. The geometric fiber $R_{\bar{t}}$ is the unipotent radical of $P_{\bar{t}}$ for each $t \in \operatorname{Spec}(\mathcal{A})$. If $P=P(\phi)$ for an $\mathcal{A}$-homomorphism $\phi: \mathbf{G}_{m} \rightarrow G$, then Lie $R_{u}(P)=\bigoplus_{n>0} \mathfrak{g}(\phi ; n)$.

Recall from Section 3.3 that by a Levi subgroup scheme of $P$ we mean a closed and smooth subgroup scheme $L \subset P$ such that $L^{o}$ is reductive and such that $L_{t}$ is a Levi factor of $P_{t}$ for each $t \in \operatorname{Spec}(\mathcal{A})$. Since $\mathcal{A}$ is assumed to be local, we have:

(3.11.5). [SGA 3, Exp. XXVI, Cor. 2.3, 2.4] P contains a Levi subgroup scheme, and $P$ contains a maximal torus.

Using [SGA 3, Exp. XXVI, Prop. 1.6], we see that the conditions of (3.3.1) hold; thus $P$ is isomorphic to the semidirect product $L \ltimes R_{u}(P)$ for any Levi factor $L$ of $P$.

In fact, we can be a bit more precise regarding Levi subgroups and maximal tori, as follows:

(3.11.6). If $P=P(\phi)$ for some $\mathcal{A}$-homomorphism $\phi: \mathbf{G}_{m} \rightarrow G$, then the centralizer in $G$ of the image of $\phi$ is a Levi subgroup scheme of $P$.

Proof. Write $L$ for the centralizer of the image of $\Phi$. Then $L$ is a closed subgroup scheme of $P$, and according to (3.6.2), $L$ is smooth over $\mathcal{A}$. Using [Sp 98, Theorem 13.4.2] we see that $L$ is indeed a Levi subgroup scheme of $P$.

(3.11.7). If $T \subset P$ is a maximal torus, then $P=P(\phi)$ for some $\mathcal{A}$ homomorphism $\phi: \mathbf{G}_{m} \rightarrow T$. In particular, there is a Levi subgroup scheme $L \subset P$ which contains $T$.

Proof. If $\eta$ denotes the generic point of $\operatorname{Spec}(\mathcal{A})$, one knows that $P_{\eta}$ is the parabolic subgroup determined by some cocharacter $\phi_{0}$ of the maximal torus $T_{\eta} \subset P_{\eta}$ (see e.g. [Mc 05, Lem. 6]). Since $T$ is an $\mathcal{A}$-torus and since $\mathcal{A}$ is normal, use (3.11.2) to find an $\mathcal{A}$-homomorphism $\phi: \mathbf{G}_{m} \rightarrow T$ such that $\phi_{0}=\phi_{\eta}$. It follows from $(3.10 .2)($ a) that $P=P(\phi)$. Finally, (3.11.6) gives the required Levi subgroup scheme of $P$. 


\section{§4. Nilpotent elements and the instability parabolic over a field}

In this section, we let $\mathfrak{f}$ be an arbitrary field, and we suppose that $G$ is a $D$-standard reductive group over $\mathfrak{f}$ with Lie algebra $\mathfrak{g}$. Let $X \in \mathfrak{g}(\mathfrak{f})$ be a nilpotent element.

\subsection{Associated cocharacters}

We write $X_{*}(G)$ for the collection of $\mathfrak{f}$-homomorphisms $\mathbf{G}_{m} \rightarrow G$. If $\Psi \in X_{*}(G)$, recall that - as in 3.11 - we may write $\mathfrak{g}=\bigoplus_{n \in \mathbf{Z}} \mathfrak{g}(\Psi ; n)$ where we regard the Lie algebra $\mathfrak{g}$ as a $G$-module via the adjoint representation.

A cocharacter $\Psi \in X_{*}(G)$ is said to be associated with $X$ (see [Ja 04, $\S 5])$ if the following conditions hold:

(A1) $X \in \mathfrak{g}(\Psi ; 2)$, and

(A2) there is a maximal torus $S$ of $C_{G}(X)$ such that $\Psi \in X_{*}\left(L_{1}\right)$ where $L=C_{G}(S)$ and $L_{1}=(L, L)$ is its derived group.

By regarding the nilpotent element $X$ as an unstable vector in the $G$ representation $\mathfrak{g}$ and using the notions of optimal cocharacters and the instability parabolic due to Kempf and to Rousseau, one finds:

(4.1.1). Let $X \in \mathfrak{g}$ be nilpotent.

(a) There is a cocharacter $\Psi$ associated with $X$.

(b) If $\Psi$ is associated to $X$, then $C_{G}(X) \subset P(\Psi)$.

(c) The unipotent radical of $C=C_{G}(X)$ is defined over $F$, and is an $\mathfrak{f}$-split unipotent group.

(d) If the cocharacter $\Psi$ is associated with $X$, then $L=C \cap C_{G}\left(\Psi\left(\mathbf{G}_{m}\right)\right)$ is a Levi factor of $C$.

(e) If $\Psi, \Phi \in X_{*}(G)$ are associated with $X$, then $\Psi=\operatorname{Int}(x) \circ \Phi$ for a unique $x \in U(K)$.

(f) The parabolic subgroups $P(\Psi)$ for cocharacters $\Psi$ associated with $X$ all coincide.

Proof. In the "geometric case" - when $\mathfrak{f}$ is algebraically closed - (a) is essentially a consequence of Pommerening's - and more recently, Premet's proof of the Bala-Carter theorem; Premet's proof [Pr 03] avoids case analysis and uses results of geometric invariant theory due to Kempf and Rousseau. Working over any ground field $\mathfrak{f},(\mathrm{a})$ is in [Mc 04, Theorem 26]. Now (b) is [Ja 04, Prop. 5.9]. (c) and (e) follow from parts (3) and (4) of [Mc 05, Prop/Defn 21]. (d) is essentially a consequence of results in [Pr 03]; see [Mc 04, Cor. 20 and Cor. 29]. Finally, (f) is [Mc 05, Prop/Defn 21(5)]. 
We write $P_{X}$ for the common parabolic subgroup of part (f) of (4.1.1); we say that $P_{X}$ is the instability parabolic subgroup attached to $X$.

\subsection{The stabilizer of the line through $X$}

Let $N_{G}(X) \subset G$ be the stabilizer of the line $[X] \in \mathbf{P}(\mathfrak{g})$, where $\mathbf{P}(\mathfrak{g})$ denotes the projective $\mathfrak{f}$-variety formed from the vector space $\mathfrak{g}$. Then:

(4.2.1). [Mc 04, Lem. 23] $N_{G}(X)$ is a smooth $\mathfrak{f}$-subgroup of $G$.

Of course, any cocharacter of $G$ associated to $X$ is a cocharacter of $N_{G}(X)$. A more precise version of (4.1.1)(a) is as follows:

(4.2.2). [Mc 04, Lem. 25] Let $S$ be a maximal torus of $N_{G}(X)$. Then there is a unique cocharacter of $S$ which is associated to $X$.

\subsection{Almost associated cocharacters}

Let $P_{X}$ be the instability parabolic subgroup attached to $X$, let $\Phi$ be a cocharacter of $G$, and let $\mathfrak{f}_{\text {sep }}$ be a separable closure of $\mathfrak{f}$. We say that $\Phi$ is almost associated to $X$ provided that $\operatorname{Int}(g) \circ \Phi$ is a cocharacter of $G_{/ \mathfrak{f}_{\mathrm{sep}}}$ associated to $X$ for some $g \in P_{X}\left(\mathfrak{f}_{\text {sep }}\right)$.

(4.3.1). Let $S \subset P_{X}$ be a maximal torus. Then there is a unique cocharacter $\Phi$ of $S$ which is almost associated to $X$. The cocharacter $\Phi$ is associated to $X$ if and only if $S$ contains a maximal torus of $N_{G}(X)$.

Proof. For the existence of $\Phi$, let $S_{1}$ be a maximal torus of $N_{G}(X)$, and let $S_{0}$ be a maximal torus of $P$ containing $S_{1}$. Then $S$ and $S_{1}$ are maximal tori of $P$ and hence are conjugate by an element $g \in P\left(\mathfrak{f}_{\text {sep }}\right)$. If $\Psi$ is the cocharacter of $S_{1}$ associated to $X$, then $\operatorname{Int}(g) \circ \Psi$ is a cocharacter of $S$ which is almost associated to $X$, as required.

We now argue the uniqueness. Since $\Phi$ is $P\left(\mathfrak{f}_{\text {sep }}\right)$-conjugate to a cocharacter associated to $X$, one knows that $\Phi$ is an optimal cocharacter for the unstable vector $X$ in the sense of geometric invariant theory; cf. [Mc 04, §3]. Thus the unicity is a consequence of the result of Kempf and of Rousseau; cf. [Mc 04, Prop. 13(4)].

The remaining assertion is clear.

(4.3.2). If the cocharacters $\Phi, \Psi$ are almost associated to $X$, then

$$
\sum_{j \geq 2} \mathfrak{g}(\Psi ; j)=\sum_{j \geq 2} \mathfrak{g}(\Phi ; j) .
$$


Proof. Indeed, we have $\Phi=\operatorname{Int}(g) \circ \Psi$ for some $g \in P\left(\mathfrak{f}_{\text {sep }}\right)$, so the assertion follows from the fact that $\sum_{j \geq 2} \mathfrak{g}(\Psi ; j)$ is $\operatorname{Ad}(P)$-stable.

We have:

(4.3.3). Let $\Psi$ be a cocharacter of $G$ which is almost associated to $X$.

(a) The $\operatorname{Ad}\left(P_{X}\right)$-orbit of $X$ is dense in $\sum_{j \geq 2} \mathfrak{g}(\Psi ; j)$.

(b) Write $X=\sum_{j \geq 2} X_{j}$ with $X_{j} \in \mathfrak{g}(\Psi ; j)$. Then $X$ is $\operatorname{Ad}(P)(\mathfrak{f})$-conjugate to $X_{2}$, and $\Psi$ is a cocharacter associated to $X_{2}$.

Proof. (a) follows by combining (4.3.2) with [Ja 04, Prop. 5.9(c)].

The conjugacy statement of (b) follows from [Mc 04, Prop. 34]. It is then clear that $\Psi$ is almost associated to $X_{2}$. Since $\Psi$ is a cocharacter of a maximal torus of $N_{X_{2}}$, it follows from (4.3.1) that $\Phi$ is associated to $X_{2}$.

In particular, (4.3.3)(b) implies:

(4.3.4). If the cocharacter $\Psi$ is almost associated with $X$ and if $X \in$ $\mathfrak{g}(\Psi ; 2)$, then $\Psi$ is associated with $X$.

\subsection{The Bala-Carter theorem}

For a $D$-standard reductive group over a field $\mathfrak{f}$, the geometric nilpotent orbits - i.e. the nilpotent orbits of $G_{/ \mathfrak{f}_{\text {sep }}}$ - are described by the Bala-Carter theorem. Let us suppose that $\mathfrak{f}=\mathfrak{f}_{\text {sep }}$.

Recall that a parabolic subgroup $P \subset G$ is distinguished if

$$
\operatorname{dim} P / U=\operatorname{dim} U /(U, U)+\operatorname{dim} Z
$$

where $U$ is the unipotent radical of $P$, and $Z$ is the center of $G$. A nilpotent element $X \in \mathfrak{g}$ is said to be distinguished if a maximal torus of $C=C_{G}(X)$ is central in $C$; if $X$ is distinguished, then the instability parabolic subgroup $P_{X}$ is distinguished.

Each parabolic subgroup has an open orbit - known as the Richardson orbit - on Lie $R_{u} P$; any element of this orbit is known as a Richardson element for $P$.

We have the following important theorem: 
(4.4.1). (The Bala-Carter theorem) Let $L$ be a Levi factor of a parabolic subgroup of $G$, and let $P \subset L$ be a distinguished parabolic subgroup of $L$. The map which associates to $(L, P)$ the $G$-orbit of a Richardson element for $P$ determines a bijection between the $G$-orbits of such pairs $(L, P)$ and the $G$-orbits on nilpotent elements in $\operatorname{Lie}(G)$.

This theorem was originally proved by Bala and Carter in the case where $p$ is "very large". Pommerening gave a proof in good characteristic, using some case analysis in a few situations. Premet [Pr 03] gave recently a short and conceptual proof of this theorem. See also [Ja 04, §4].

If the orbit of a nilpotent element $X \in \operatorname{Lie}(G)$ corresponds via the BalaCarter theorem to the pair $(L, P)$, then the $G$-orbit of $(L, P)$ - or, abusing terminology somewhat, just the pair $(L, P)$ - is said to be the Bala-Carter datum for $X$.

(4.4.2). Let $\mathfrak{f}$ and $\mathfrak{f}^{\prime}$ be algebraically closed fields of the same characteristic, suppose that $G$ and $G^{\prime}$ are D-standard reductive groups respectively over $\mathfrak{f}$ and $\mathfrak{f}^{\prime}$ with identical root data, let $X \in \operatorname{Lie}(G)$ and $X^{\prime} \in \operatorname{Lie}\left(G^{\prime}\right)$ be nilpotent elements with the same Bala-Carter datum, and let $C, C^{\prime}$ be their respective centralizers. Then:

(1) the root datum of a Levi factor of $C$ identifies with the root datum of a Levi factor of $C^{\prime}$, and

(2) $C / C^{o} \simeq C^{\prime} / C^{\prime o}$.

Proof. Indeed, we may choose an algebraically closed field $\mathfrak{f}^{\prime \prime}$ containing both $\mathfrak{f}$ and $\mathfrak{f}^{\prime}$. We thus see that it suffices to prove the result when $\mathfrak{f} \subset \mathfrak{f}^{\prime}$.

But then the Bala-Carter theorem implies that $X$ and $X^{\prime}$ are conjugate by an element of $G^{\prime}$, and the result is immediate.

\section{§5. Nilpotent sections and the instability parabolic over $\mathcal{A}$}

In this section, let $G$ be a $D$-standard (see Section 3) reductive group scheme over $\mathcal{A}$.

\subsection{Equidimensional nilpotent sections}

Let $X \in \mathfrak{g}(\mathcal{A})$ be a section of the Lie algebra $\mathfrak{g}=\operatorname{Lie}(G)$, and let $C_{G}(X)=\operatorname{Stab}_{G}(X)$ be the centralizer of this section; cf. Section 2.3. On base change, the group $C_{G}(X)_{t}$ is the centralizer of $X(t)$ in the algebraic group $G_{t}$ for each point $t$ of $\operatorname{Spec}(\mathcal{A})$; according to (3.9.2), each group 
$C_{G}(X)_{t}$ is smooth over $k(t)$. In general, however, the group scheme $C_{G}(X)$ will not be smooth - or even flat - over $\mathcal{A}$.

We say that $X$ is nilpotent if the value of $X$ at the generic point $\eta \in$ $\operatorname{Spec}(\mathcal{A})$ is nilpotent - i.e. if $X(\eta) \in \mathfrak{g}(K)$ is nilpotent.

(5.1.1). If $X$ is nilpotent, then also the value $X(t) \in \mathfrak{g}(k(t))$ is nilpotent for each point $t \in \operatorname{Spec}(\mathcal{A})$.

Proof. Let $\lambda$ denote the regular representation of $G$ on $\mathcal{A}[G]$. Since $G$ is reductive, it is by definition smooth - and in particular, flat - over $\mathcal{A}$. Since the coordinate algebra $\mathcal{A}[G]$ is a flat $\mathcal{A}$-module, we may regard $\mathcal{A}[G]$ as a subring of $K[G]$. Since $X$ is nilpotent, the operator $a(\eta)=$ $d \lambda(X(\eta)): K[G] \rightarrow K[G]$ is locally nilpotent; i.e. for each $f \in K[G]$, we have $a(\eta)^{N(f)} f=0$ for some $N(f)>0$.

Let $t \in \operatorname{Spec}(\mathcal{A})$, and consider the localization $\mathcal{A}_{t}=\mathcal{A}_{\mathfrak{p}}$ where $\mathfrak{p} \subset \mathcal{A}$ is the prime ideal that "is" the point $t$. Then we have $\mathcal{A}[G] \subset \mathcal{A}_{t}[G] \subset K[G]$, and $a(\eta)$ restricts to a locally nilpotent endomorphism $a$ of $\mathcal{A}[G]$ and of $\mathcal{A}_{t}[G]$. Since $k(t)$ is a quotient of $\mathcal{A}_{t}$, it follows that $a(t)=d \lambda(X(t))$ : $k(t)[G] \rightarrow k(t)[G]$ is locally nilpotent, so that $X(t)$ is indeed nilpotent as required.

We say that a nilpotent section $X \in \mathfrak{g}(\mathcal{A})$ is equidimensional if $\operatorname{dim} C_{G}(X)_{t}$ is constant for each $t \in \operatorname{Spec}(\mathcal{A})$. For example, if $G=\mathrm{GL}_{3}$ and $\mathcal{A}$ is a discrete valuation ring with uniformizing element $\pi$, consider the nilpotent sections

$$
X_{1}=\left(\begin{array}{ccc}
0 & 1 & 0 \\
0 & 0 & 1 \\
0 & 0 & 0
\end{array}\right), X_{2}=\left(\begin{array}{ccc}
0 & 1 & 0 \\
0 & 0 & \pi \\
0 & 0 & 0
\end{array}\right) \in \mathfrak{g l}_{3}(\mathcal{A})
$$

Then $X_{1}$ is equidimensional, while $X_{2}$ is not.

(5.1.2). Let $\eta, s \in \operatorname{Spec}(\mathcal{A})$ be respectively the generic point and the closed point. If

$$
\operatorname{dim} C_{G}(X)_{\eta}=\operatorname{dim} C_{G}(X)_{s}
$$

then $X$ is equidimensional.

Proof. Since $C_{G}(X)$ is the fiber product $G \times{ }_{\mathfrak{g}} \operatorname{Spec}(\mathcal{A})$, it is a scheme of finite type over $\mathcal{A}$. The assertion now follows from (2.5.2). 


\subsection{Smoothness}

If $\mathcal{L}$ is a free $\mathcal{A}$-module of finite rank $d$, we can regard $\mathcal{L}$ as an $\mathcal{A}$-scheme isomorphic to $\mathbf{A}^{d}$. Moreover, we may consider the $\mathcal{A}$-scheme $\mathbf{P}(\mathcal{L})$ given for each commutative $\mathcal{A}$-algebra $\Lambda$ by

\section{$\mathbf{P}(\mathcal{L})(\Lambda)=$ set of those $\Lambda$-direct summands of $\mathcal{L} \otimes_{\mathcal{A}} \Lambda$ having rank 1.}

Then $\mathbf{P}(\mathcal{L})$ is isomorphic to $\mathbf{P}^{d-1}$. For each $s \in \operatorname{Spec}(\mathcal{A})$, the scheme $\mathbf{P}(\mathcal{L})_{s}$ obtained by base-change is just the projective space of the $k(s)$-vector space $\mathcal{L}_{s}=\mathcal{L} \otimes_{\mathcal{A}} k(s)$.

We are going to consider the $\mathcal{A}$-schemes $\mathfrak{g}$ and $\mathbf{P}(\mathfrak{g})$ where $\mathfrak{g}=\operatorname{Lie}(G)$. Of course, $G$ acts on $\mathfrak{g}$ by the adjoint representation. If $Y \in \mathfrak{g}(A)$, we write $C_{G}(Y)$ for the stabilizer $\operatorname{Stab}_{G}(Y)$ of the sections $Y$.

The adjoint action of $G$ on $\mathfrak{g}$ determines also an action of $G$ on the projective space $\mathbf{P}(\mathfrak{g})$. If $Y \in \mathfrak{g}(\mathcal{A})$ is a section whose image in $\mathfrak{g}(k)$ is nonzero, where $k$ is the residue field of $\mathcal{A}$, then $\mathcal{A} Y$ is an $\mathcal{A}$-direct summand of $\mathfrak{g}(\mathcal{A})$, so it determines a section $[Y] \in \mathbf{P}(\mathfrak{g})(\mathcal{A})$. We write $N_{G}(Y)=$ $\operatorname{Stab}_{G}([Y])$ for this stabilizer.

Let $X \in \mathfrak{g}(\mathcal{A})$ be a non-zero equidimensional nilpotent section. Since $X$ is equidimensional, evidently $X(s) \neq 0$; thus $X$ determines a section $[X] \in \mathbf{P}(\mathfrak{g})(\mathcal{A})$.

Proposition. The subgroup schemes $C_{G}(X)$ and $N_{G}(X)$ of $G$ are smooth over $\mathcal{A}$.

Proof. For each $t \in \operatorname{Spec}(\mathcal{A})$, we know that the dimension of $N_{G}(X)_{t}$ is one more than the dimension of $C_{G}(X)_{t}$; cf. [Ja 04, §5.3]. We know from (3.9.2) that $C_{G}(X)_{t}$ is smooth, and it follows from [Mc 04, Lem. 23] that $N_{G}(X)_{t}$ is smooth. Thus the Proposition follows from (2.3.2) using equidimensionality.

\subsection{Richardson sections}

Let $X=\mathbf{A}_{/ \mathbf{Z}}^{n}$ be affine $n$-space over $\mathbf{Z}$ for some $n \geq 1$, and let $S=$ $\left\{p_{1}, \ldots, p_{n}\right\}$ be a finite set of $n$ distinct prime numbers. We regard the $p_{i}$ as points of $\operatorname{Spec}(\mathbf{Z})$, and we write $\xi$ for the generic point of $\operatorname{Spec}(\mathbf{Z})$.

Suppose that we are given an open $\mathbf{Q}$-subscheme $U_{0}$ of the generic fiber $X_{\xi}$, and that for each $p \in S$, we are given an open $\mathbf{F}_{p}$-subscheme $U_{p}$ of the fiber $X_{p}$.

For a regular function $f \in \mathbf{Z}[X]$ and a field $E$, we write $f_{E}$ for the corresponding regular function $f \otimes 1$ in $E[X]=\mathbf{Z}[X] \otimes_{\mathbf{Z}} E$. 
(5.3.1). There is a regular function $f \in \mathbf{Z}[X]$ such that

(i) the distinguished open subset of $X_{\xi}$ determined by the non-vanishing of $f_{\mathbf{Q}}$ is contained in $U_{0}$, and

(ii) for $p \in S$, the distinguished open subset of $X_{p}$ determined by the nonvanishing of $f_{\mathbf{F}_{p}}$ is contained in $U_{p}$.

Proof. Let $g \in \mathbf{Q}[X]$ be a regular function such that the distinguished open subset $D(g)$ of $X_{\xi}$ determined by the non-vanishing of $g$ lies in $U_{\xi}$. We may evidently replace $g$ by a non-zero integer multiple without changing $D(g)$; since $\mathbf{Z}[X]=\mathbf{Z}\left[T_{1}, \ldots, T_{n}\right]$ is a factorial domain, we may suppose that $g \in \mathbf{Z}[X]$ and that the image of $g$ in $\mathbf{F}_{p}[X]$ is non-zero for each prime $p$.

For $p \in S$, let $h_{p} \in \mathbf{F}_{p}[X]$ be a non-zero regular function such that the distinguished open subset $D\left(h_{p}\right)$ of $X_{p}$ determined by $h_{p}$ lies in the open subscheme $U_{p}$. Since the natural mapping $\mathbf{Z}[X] \rightarrow \prod_{p \in S} \mathbf{F}_{p}[X]$ is surjective by the Chinese Remainder Theorem, we may find $h \in \mathbf{Z}[X]$ whose image in $\mathbf{F}_{p}[X]$ is $h_{p}$ for each $p \in S$.

Now put $f=g \cdot h \in \mathbf{Z}[X]$. Then for each $p \in S$, the image $f_{\mathbf{F}_{p}}$ is non-zero; since $h_{p} \mid f_{\mathbf{F}_{p}}$, the distinguished open subset of $X_{p}$ determined by the non-vanishing of $f_{\mathbf{F}_{p}}$ is contained in $U_{p}$. Moreover, since $g \mid f=f_{\mathbf{Q}}$, the distinguished open subset of $X_{\xi}$ determined by the non-vanishing of $f_{\mathbf{Q}}$ is contained in $U_{0}$, as required.

Let now $\mathcal{A}$ be a local, normal, Noetherian domain, and suppose that $G$ is a split reductive group over $\mathcal{A}$, with split maximal torus $T$.

(5.3.2). Let $P \subset G$ be a parabolic subgroup scheme containing $T$.

(a) There is a regular function $f \in \mathcal{A}\left[\operatorname{Lie}\left(R_{u} P\right)\right]$ such that for each $t \in$ $\operatorname{Spec}(\mathcal{A})$, the distinguished open subset of the $k(t)$-scheme $\operatorname{Lie}\left(R_{u} P\right)_{t}$ determined by the non-vanishing of $f_{k(t)}$ is contained in the Richardson orbit of $P_{t}$ on $\operatorname{Lie}\left(R_{u} P\right)_{t}$.

(b) If the residue field of $\mathcal{A}$ is infinite, there is a section $X \in \operatorname{Lie}\left(R_{u} P\right)(\mathcal{A})$ such that $X(t)$ is a Richardson element for $P_{t}$ for each $t \in \operatorname{Spec}(\mathcal{A})$.

Proof. There is a split reductive group scheme $G_{0}$ over $\mathbf{Z}$ and a split maximal torus $T_{0}$ such that $G=G_{0 / \mathcal{A}}$ and $T=T_{0 / \mathcal{A}}$.

Now choose an $A$-homomorphism $\phi: \mathbf{G}_{m} \rightarrow T$ such that $P=P(\phi)$ as in (3.11.7). Since $T_{0}$ is a split torus over $\mathbf{Z}$, there is a $\mathbf{Z}$-homomorphism 
$\psi: \mathbf{G}_{m} \rightarrow T_{0}$ such that $\phi=\psi_{/ \mathcal{A}}$. Writing $P_{0}$ for the parabolic $\mathbf{Z}$-subgroup scheme of $G_{0}$ determined by $\psi$, we have $P=P_{0 / \mathcal{A}}$.

On the geometric fibers, it follows from the finiteness of the number of nilpotent $G_{0, \bar{p}}$-orbits ${ }^{5}$ in $\operatorname{Lie}\left(G_{0}\right)_{\bar{p}}$ that $P_{0, \bar{p}}$ has an open orbit (the Richardson orbit) in $\operatorname{Lie}\left(R_{u}\left(P_{0}\right)\right)_{\bar{p}}$ for each $p \in \operatorname{Spec}(\mathbf{Z})$. Using [Sp 98, Prop. 11.2.8] one knows that this open orbit is obtained by base change from an open $k(p)$-subscheme for each $p \in \operatorname{Spec}(\mathbf{Z})$.

Let $p$ denote the characteristic of the residue field of $\mathcal{A}$; if $p>0$, let $S=\{p\}$; otherwise, let $S=\emptyset$. Now use (5.3.1) applied to $X=\operatorname{Lie}\left(R_{u} P_{0}\right)$ and the set $S$ to find a regular function $f \in \mathbf{Z}\left[\operatorname{Lie}\left(R_{u} P_{0}\right)\right]$ whose image in $\mathcal{A}\left[\operatorname{Lie}\left(R_{u} P\right)\right]$ has the required properties. This proves (a).

For (b), since $k(s)=k$ is infinite, where $s \in \operatorname{Spec}(\mathcal{A})$ is the closed point, one may choose an element $Y \in \operatorname{Lie}\left(R_{u} P\right)(k)$ such that the regular function $f_{k}$ does not vanish at $Y$. Let $X \in \operatorname{Lie}\left(R_{u} P\right)(\mathcal{A})$ be any section such that $X(s)=Y$. Then evidently the value $f(X)$ is a unit in $\mathcal{A}$; it now follows from (a) that $X(t)$ is a Richardson element for $P_{t}$ for each $t \in \operatorname{Spec}(\mathcal{A})$, as required.

\subsection{Existence of equidimensional nilpotent sections}

Assume throughout this section that $\mathcal{A}$ is a normal, local, Noetherian domain with infinite residue field $k$, and that the reductive group scheme $G$ is split over $\mathcal{A}$, with split maximal torus $T \subset G$. We suppose that $G$ is $D$-standard.

Let $L \subset G$ be a Levi factor of some parabolic subgroup scheme of $G$, and suppose that $T \subset L$. We remark that $L$ itself is $D$-standard.

Since $T$ is a split torus, we may choose an isomorphism $T \simeq D_{\mathcal{A}}(X)$ where $X=X(T)$ is the free Abelian group $\mathbf{Z}^{r}$; then $X(T)$ identifies with the group of characters $\operatorname{Hom}_{\mathcal{A}}\left(T, \mathbf{G}_{m}\right)$. From the roots of $L$ with respect to $T$, choose a system of positive roots $R^{+} \subset X$ and a basis of the roots $\Pi \subset R^{+}$.

Now write $\operatorname{Der}(L)=L^{\prime}$ for the derived subgroup scheme as in 3.7, and write $T^{\prime}$ for the maximal split torus of $L^{\prime}$ contained in $T$; cf. (3.7.2). Then $X\left(T^{\prime}\right)$ contains $\mathbf{Z} R$ as a subgroup of finite index.

(5.4.1). Let $Q_{0} \subset L_{t}$ be a distinguished parabolic subgroup containing $T_{t}$ for some $t \in \operatorname{Spec}(\mathcal{A})$.

\footnotetext{
${ }^{5}$ That finiteness is true in all characteristics, though the proof in bad characteristic is "case-by-case" at present. See [Ja 04, §2.8].
} 
(a) There is a parabolic subgroup scheme $Q \subset L$ such that $Q_{0}=Q_{t}$.

(b) $Q_{x}$ is a distinguished parabolic subgroup of $L_{x}$ for every $x \in \operatorname{Spec}(\mathcal{A})$.

(c) Let $I \subset \Pi$ be defined by the condition $\alpha \in I \Longleftrightarrow \operatorname{Lie}\left(Q_{0}\right)_{-\alpha} \neq 0$. Then there is a unique $\mathcal{A}$-homomorphism $\phi: \mathbf{G}_{m} \rightarrow T$ such that $\langle\alpha, \phi\rangle=2$ for $\alpha \in \Pi-I$ and $\langle\alpha, \phi\rangle=0$ for $\alpha \in I$. Moreover, $Q$ is the parabolic subgroup of $L$ determined by $\phi$.

Proof. Since $L$ is $D$-standard, the characteristic of $k(x)$ is good for the derived group of $L_{x}$ for every $x \in \operatorname{Spec}(\mathcal{A})$. It then follows from [Ja 04, Lem. 5.2] that the homomorphism $\mathbf{Z} R \rightarrow \mathbf{Z}$ given by the rule in (c) determines an $\mathcal{A}$-homomorphism $\phi: \mathbf{G}_{m} \rightarrow T^{\prime}$.

Let $Q$ be the parabolic subgroup of $L$ determined by $\phi$. (a) is then clear, and (b) follows from [Ja 04, $§ 4.10(2)]$.

(5.4.2). Let $\phi: \mathbf{G}_{m} \rightarrow T$ be the cocharacter of (5.4.1)(c). There is a section

$$
X \in \operatorname{Lie}(L)(\phi ; 2)(\mathcal{A})
$$

such that

(a) $X(t)$ is a Richardson element for $Q_{t}$,

(b) $\phi_{t}$ is associated with $X(t)$, and

(c) the Bala-Carter datum of $X(t)$ is $\left(L_{t}, Q_{t}\right)$.

for each $t \in \operatorname{Spec}(\mathcal{A})$. Moreover, $X$ is an equidimensional nilpotent section of $\operatorname{Lie}(G)$.

Proof. Since the residue field of $\mathcal{A}$ is assumed to be infinite, we may use (5.3.2) to find a section $Y \in \operatorname{Lie}\left(R_{u} Q\right)(\mathcal{A})$ such that $Y(t)$ is a Richardson element for $Q_{t}$ for each $t \in \operatorname{Spec}(\mathcal{A})$.

It follows from [Ja 04, Lem. 5.2 and Lem. 5.3] that $\phi_{t}$ is almost associated with $X(t)$ for each $t \in \operatorname{Spec}(\mathcal{A})$.

Since $Q$ is the parabolic subgroup determined by $\phi$, we know that

$$
\operatorname{Lie}\left(R_{u} Q\right)=\sum_{i \geq 1} \operatorname{Lie}(L)(\phi ; i) .
$$

Thus, we may write $Y=\sum_{i \geq 1} Y_{i}$ with $Y_{i} \in \operatorname{Lie}(L)(\phi: i)(\mathcal{A})$.

It follows from (4.3.3) that for each $t \in \operatorname{Spec}(\mathcal{A})$, the element $Y_{2}(t)$ is Richardson for $Q_{t}$, and the cocharacter $\phi_{t}$ is associated with $Y_{2}(t)$; in particular, if we set $X=Y_{2}$ then (a), (b), and (c) hold for $X$. 
Write $P$ for the parabolic subgroup scheme $P(\phi) \subset G$. Since $\phi_{t}$ is associated with $X(t)$ for each $t \in \operatorname{Spec}(\mathcal{A})$, we know $P_{t}$ to be the instability parabolic of $X(t)$, so that - by (4.1.1) - we have $C_{G}(X)_{t} \subset P_{t}$ for each point $t$ of $\operatorname{Spec}(\mathcal{A})$. Now, the $P_{t}$-orbit of $X(t)$ is dense in $\sum_{j \geq 2} \mathfrak{g}(\phi ; j)_{t}$ by (4.3.3). It follows that the centralizer of $X$ in $P$ has constant dimension on the fibers of $\operatorname{Spec}(\mathcal{A})$, so that $X$ is indeed equidimensional.

Theorem. Let $t \in \operatorname{Spec}(\mathcal{A})$ and let $Y \in \mathfrak{g}(k(\bar{t}))$ be a nilpotent element. Then there is a Levi subgroup scheme $L$ of a parabolic subgroup scheme of $G$, an $\mathcal{A}$-homomorphism $\Phi: \mathbf{G}_{m} \rightarrow L$, and a nilpotent section $X \in$ $\operatorname{Lie}(L)(\Phi ; 2)(\mathcal{A})$ for which the following conditions hold:

(a) $X$ is an equidimensional nilpotent section of $\mathfrak{g}$,

(b) $X(t)$ is $G_{\bar{t}}$-conjugate to $Y$,

(c) for each $u \in \operatorname{Spec}(\mathcal{A})$, the Bala-Carter datum of $X(\bar{u})$ is $\left(L_{\bar{u}}, Q_{\bar{u}}\right)$, where $Q$ is the parabolic subgroup scheme $P_{L}(\Phi)$ of $L$ determined by $\Phi$.

(d) $P_{u}$ is the instability parabolic of $G_{u}$ determined by $X(u)$ for each $u \in$ $\operatorname{Spec}(\mathcal{A})$, where $P=P_{G}(\Phi)$ is the parabolic subgroup scheme of $G$ determined by $\Phi$.

In particular, $\Phi_{u}$ is a cocharacter of $G_{u}$ associated with $X(u)$ for each $u \in$ $\operatorname{Spec}(\mathcal{A})$.

Proof. Recall that $T$ is a fixed split maximal torus of $G$. Suppose that $\left(L_{0}, Q_{0}\right)$ is the Bala-Carter datum of $Y$; thus $L_{0}$ is a Levi subgroup of a parabolic subgroup of $G_{t}, Y$ is distinguished in $\operatorname{Lie}\left(L_{0}\right)$, and $Q_{0}$ is a distinguished parabolic subgroup of $L_{0}$. Since we work up to geometric conjugacy, we may as well suppose that $L_{0}$ is defined over $k(t)$, that $L_{0}$ contains $T_{t}$, and that $T_{t}$ contains a maximal torus of the centralizer in $G$ of $Y$. This last condition shows that $L_{0}$ is the centralizer of the image of some cocharacter of $T_{t}$. Since $T$ is a split torus, this cocharacter arises by base change from an $\mathcal{A}$-homomorphism $\Phi: \mathbf{G}_{m} \rightarrow T$; in view of (3.11.6), there is a Levi subgroup scheme $L$ of a parabolic subgroup scheme of $G$ for which $L_{t}=L_{0}$. Now use (5.4.1) to see that $Q=P_{L}(\Phi)$ is a distinguished parabolic subgroup scheme of $L$ for which $Q_{t}$ is $L_{\bar{\eta}}$ conjugate to $Q_{0}$. Finally, use (5.4.2) to find an equidimensional nilpotent section $X \in \operatorname{Lie}(L)(\Phi ; 2)(\mathcal{A})$ for which $X(u)$ has Bala-Carter datum $\left(L_{u}, Q_{u}\right)$ for each $u \in \operatorname{Spec}(\mathcal{A})$. Then $X(t)$ is $G_{\bar{t}}$-conjugate to $Y$. Thus (a), (b) and (c) hold. 
By (5.4.2), $\Phi_{u}$ is a cocharacter of $G_{u}$ associated with $X(u)$ for each $u \in \operatorname{Spec}(\mathcal{A})$. Denoting by $P$ the parabolic subgroup scheme $P_{G}(\phi)$, we conclude that $P_{u}$ is the instability parabolic of $X(u)$ for each $u \in \operatorname{Spec}(\mathcal{A})$; thus (d) holds as well.

\subsection{The instability parabolic of $X$}

Let $X \in \mathfrak{g}(\mathcal{A})$ be an equidimensional nilpotent section. Let $\eta \in \operatorname{Spec}(\mathcal{A})$ be the generic point, and let $P_{0} \subset G_{\eta}$ by the instability parabolic subgroup determined by $X(\eta)$.

Proposition. There is a unique $\mathcal{A}$-parabolic subgroup scheme $P \subset G$ such that $P_{0}=P_{\eta}$.

Proof. Unicity follows from (3.10.2)(a). For existence, first suppose that $\mathcal{A}$ is a discrete valuation ring. In that case the conclusion of the Proposition is a consequence of (3.10.3).

Since the scheme $\underline{P a r}$ of parabolic subgroups of $G$ is projective (3.10.1), the Proposition now follows in the general case from (2.6.3).

Remark. The conclusion of the Proposition has already been observed for the nilpotent sections obtained using Theorem 5.4.

\section{6. Étale local existence of associated cocharacters over $\mathcal{A}$}

With notation as before, write $P \subset G$ be the parabolic subgroup scheme for which $P_{\eta}$ is the instability parabolic of $X(\eta)$.

(5.6.1). (i) There is an $\mathcal{A}$-homomorphism $\Phi: \mathbf{G}_{m} \rightarrow P$ such that the cocharacter $\Phi_{\eta}$ is almost-associated with $X(\eta)$.

(ii) For each $t \in \operatorname{Spec}(\mathcal{A})$, the $P_{t}$-orbit of $X(t)$ is separable and dense in $\sum_{i \geq 2} \mathfrak{g}(\Phi ; i)_{t}$

Proof. Using (3.11.5), we choose a maximal torus $T \subset P$. Let $\Phi_{0}$ be the unique cocharacter of $T_{\eta}$ which is almost-associated to $X(\eta)$. It follows from (3.11.2) that there is an $\mathcal{A}$-homomorphism $\Phi: \mathbf{G}_{m} \rightarrow T$ inducing $\Phi_{0}$ on base-change; this proves (i).

We now prove (ii). Since $\Phi_{\eta}$ is almost associated with $X(\eta)$, the $P_{\eta^{-}}$ orbit of $X(\eta)$ is dense in $\sum_{i \geq 2} \mathfrak{g}(\Psi ; j)_{\eta}$ by (4.3.3). In particular, $X$ may be regarded as an $\mathcal{A}$-section of $\sum_{i \geq 2} \mathfrak{g}(\Psi ; j)$ and so $X(t)$ is a section of $\sum_{i \geq 2} \mathfrak{g}(\Psi ; j)_{t}$. 
Write $d$ for the $\mathcal{A}$-rank of the free $\mathcal{A}$-module $\sum_{i \geq 2} \mathfrak{g}(\Psi ; j)$. Since the centralizer of $X(\eta)$ in $G_{\eta}$ is contained in $P_{\eta}$, we have by assumption that

$$
\operatorname{dim} P_{\eta}-\operatorname{dim} C_{G}(X)_{\eta}=d
$$

Now, we certainly have $\operatorname{dim} C_{P}(X)_{t} \leq \operatorname{dim} C_{G}(X)_{t}$. Since the $P_{t}$-orbit of $X(t)$ lies in $\sum_{i \geq 2} \mathfrak{g}(\Psi ; j)_{t}$, and since $X$ is equidimensional, this orbit has dimension

$$
\operatorname{dim} P_{t}-\operatorname{dim} C_{P}(X)_{t} \geq \operatorname{dim} P_{t}-\operatorname{dim} C_{G}(X)_{t}=d
$$

For dimension reasons, we conclude that the $P_{t}$-orbit of $X(t)$ is dense in $\sum_{i>2} \mathfrak{g}(\Psi ; i)_{t}$. Since $C_{G}(X)_{t}$ is smooth (3.9.2), the dimension of the centralizer of $X(t)$ in the Lie algebra $\mathfrak{g}_{t}$ coincides with $\operatorname{dim} C_{G}(X)_{t}$. It follows that the dimension of the centralizer of $X(t)$ in the Lie algebra Lie $(P)_{t}$ must coincide with $\operatorname{dim} C_{P}(X)_{t}$, so that the $P_{t}$-orbit of $X(t)$ is indeed separable.

Let $M$ be a free $\mathcal{A}$-module of finite rank, and write $M_{\eta}=M \otimes_{\mathcal{A}} K$.

(5.6.2). If $N, N^{\prime} \subset M$ are $\mathcal{A}$-direct summands of $M$, then $N=N^{\prime}$ if and only if $N_{\eta}=N_{\eta}^{\prime}$.

Proof. Indeed, for any $\mathcal{A}$-direct summand $L$ of $M$, we have $L=L_{\eta} \cap M$. Thus $N_{\eta}=N_{\eta}^{\prime}$ indeed implies that $N=N^{\prime}$; the other implication is even simpler.

Proposition. Let $P_{1} \subset G$ be the $\mathcal{A}$-parabolic subgroup scheme for which $P_{1, \eta}$ is the instability parabolic of $X(\eta)$. Let $\Psi: \mathbf{G}_{m} \rightarrow P_{1}$ be an $\mathcal{A}$-homomorphism such that $\Psi_{\eta}$ is almost associated to $X(\eta)$. Then

(i) $P_{1, t}$ is the instability parabolic of $X(t)$ for each $t \in \operatorname{Spec}(\mathcal{A})$.

(ii) $\Psi_{t}$ is almost associated to $X(t)$ for each $t \in \operatorname{Spec}(\mathcal{A})$.

(iii) There is a finite, étale, local extension $\mathcal{B} \supset \mathcal{A}$ and a section $g \in P_{1}(\mathcal{B})$ such that if we put

$$
\Phi=\operatorname{Int}(g) \circ \Psi: \mathbf{G}_{m} \longrightarrow P_{1},
$$

then $\Phi_{t}$ is a cocharacter associated to $X(t)$ for each $t \in \operatorname{Spec}(\mathcal{A})$. 
Proof. Since $P_{1, \eta}=P_{1}(\Psi)_{/ \eta}$, it follows from the uniqueness assertion in Proposition 5.5 that $P_{1}=P(\Psi)$. Also notice that (i) and (ii) are consequences of (iii); we will just prove (iii).

The statement is unchanged if we replace $\mathcal{A}$ by a finite, étale, local extension; thus, we may and will suppose that $G$ is split, say with split maximal torus $T$.

Using Theorem 5.4, we locate a Levi subgroup scheme $L$ of a parabolic subgroup scheme of $G$, an $\mathcal{A}$-homomorphism $\Phi: \mathbf{G}_{m} \rightarrow L$ and a section $Y \in \operatorname{Lie}(L)(\Phi ; 2)(\mathcal{A})$ such that

- $Y$ is an equidimensional nilpotent section of $\operatorname{Lie}(G)$,

- $Y(\eta)$ is $G_{\bar{\eta}^{-}}$-conjugate to $X(\eta)$,

- $\left(L_{u}, Q_{u}\right)$ is the Bala-Carter datum of $Y(u)$ for each $u \in \operatorname{Spec}(\mathcal{A})$, where $Q=P_{L}(\Phi)$ is the parabolic subgroup scheme of $L$ determined by $\Phi$, and

- for every $u \in \operatorname{Spec}(\mathcal{A}), P_{u}$ is the parabolic subgroup of $G_{u}$ associated with $Y(u)$ and $\Phi_{u}$ is a cocharacter of $G_{u}$ associated with $Y(u)$, where $P=P_{G}(\Phi)$ is the parabolic subgroup scheme of $G$ determined by $\Phi$.

We may evidently suppose that $Q$ contains the split maximal torus $T$ of $G$.

We know that $P_{1, \eta}$ and $P_{\eta}$ are $G_{\bar{\eta}}$-conjugate. After possibly replacing $\mathcal{B}$ by a finite étale, local extension, we may suppose that $P_{1, \eta}$ and $P_{\eta}$ are conjugate by an element in $G(k(\eta))$. Using [SGA 3, Exp. XXVI, Cor. 5.5 (i)] we see that $P_{1}$ and $P$ are $G(\mathcal{A})$-conjugate; thus we may and will suppose that $P_{1}=P$. Then both cocharacters $\Phi_{\eta}$ and $\Psi_{\eta}$ are almost associated with $X(\eta)$.

It follows that $\sum_{i \geq 2} \mathfrak{g}(\Psi ; i)_{\eta}=\sum_{i \geq 2} \mathfrak{g}(\Phi ; i)_{\eta}$; using (5.6.2), we can now conclude that $\sum_{i \geq 2} \mathfrak{g}(\Psi ; i)=\sum_{i \geq 2} \mathfrak{g}(\Phi ; i)$.

By (5.6.1) the $P_{t}$-orbits of $X(t)$ and of $Y(t)$ are separable and dense in

$$
\sum_{j \geq 2} \mathfrak{g}(\Phi ; j)_{t}=\sum_{j \geq 2} \mathfrak{g}(\Psi ; j)_{t}
$$

for each point $t$ of $\operatorname{Spec}(\mathcal{A})$. Using (2.4.2) we may find a finite, étale, local extension $\mathcal{B}$ of $\mathcal{A}$ such that $X$ and $Y$ are conjugate by an element of $P(\mathcal{B})$; we may and will replace $\mathcal{A}$ with $\mathcal{B}$ so that $X$ and $Y$ are conjugate by an element of $P(\mathcal{A})$. Thus, we may and will suppose that $X=Y$.

Since the centralizers of $\Phi$ and of $\Psi$ are Levi subgroup schemes of $P$ (3.11.6), we may find maximal tori $T_{1}, T_{2} \subset P$ such that $\Phi$ factors through 
the inclusion of $T_{1}$ in $P$ and such that $\Psi$ factors through the inclusion of $T_{2}$ in $P$. Since $T_{1}$ and $T_{2}$ are locally conjugate for the étale topology of $P$ [SGA 3, Exp. XII, Thm. 1.7], after replacing $\mathcal{A}$ by a finite, étale, local extension, the maximal tori $T_{1}$ and $T_{2}$ are conjugate by an element of $P(\mathcal{A})$. Thus we may suppose that $T_{1}=T_{2}$; but then $\Phi_{\eta}=\Psi_{\eta}$ by (4.3.1). It now follows that $\Phi=\Psi$. But then one knows for each $t \in \operatorname{Spec}(\mathcal{A})$ that $\Phi_{t}=\Psi_{t}$ is associated with $Y(t)=X(t)$ and the proof is complete.

\subsection{Maximal tori and Levi factors}

We are going to prove in this section the main Theorem regarding the existence of a Levi factor of the centralizer of an equidimensional nilpotent section. We first require a preliminary observation.

Let $H$ be a smooth group scheme over $\mathcal{A}$. For $t \in \operatorname{Spec}(\mathcal{A})$, let $\rho_{r}(t)=$ $\rho_{r, H}(t)$ be the dimension of a maximal torus of the $k(\bar{t})$-group $H_{\bar{t}}$ for some (hence any) geometric point $\bar{t}$ above $t$.

\section{(5.7.1). The following are equivalent:}

(a) The function $\rho_{r}$ is constant on $\operatorname{Spec}(\mathcal{A})$.

(b) $\rho_{r}(s)=\rho_{r}(\eta)$ where $s$ and $\eta$ are respectively the closed point and the generic point of $\operatorname{Spec}(\mathcal{A})$.

(c) Locally in the étale topology, $H$ has a maximal torus.

Proof. The equivalence of (a) and (c) follows from [SGA 3, Exp. XII, Thm. 1.7(b)], while the equivalence of (a) and (b) follows from the lower semi-continuity of $\rho_{r}$ on $\operatorname{Spec}(\mathcal{A})$; cf. loc. cit. Thm. 1.7(a).

TheOREM. Let $G$ be a D-standard reductive group scheme over $\mathcal{A}$, let $X \in \mathfrak{g}(\mathcal{A})$ be an equidimensional nilpotent section, let $C=C_{G}(X)$, and let $N=N_{G}(X)$.

(a) There is a finite, étale, local extension $\mathcal{B} \supset \mathcal{A}$ and a $\mathcal{B}$-homomorphism $\phi: \mathbf{G}_{m} \rightarrow G_{/ \mathcal{B}}$ such that the cocharacter $\phi_{t}$ of $G_{t}$ is associated to $X(t)$ for each $t \in \operatorname{Spec}(\mathcal{B})$.

(b) $C$ has a Levi factor locally in the étale topology of $\operatorname{Spec}(\mathcal{A})$.

(c) $C$ has a maximal torus locally in the étale topology of $\operatorname{Spec}(\mathcal{A})$.

(d) $N$ has a maximal torus locally in the étale topology of $\operatorname{Spec}(\mathcal{A})$. 
Proof. Let $P$ be the $\mathcal{A}$-parabolic subgroup scheme of $G$ for which $P_{\eta}$ is the instability parabolic for $X(\eta)$; see Proposition 5.5. Now, (a) has been proved already in Proposition 5.6.

In order to prove (b), (c) and (d), we may and will replace $\mathcal{A}$ by a finite, étale, local extension; thus we may suppose by part (a) that $\phi: \mathbf{G}_{m} \rightarrow G$ is an $\mathcal{A}$-homomorphism for which $\phi_{t}$ is associated with $X(t)$ for all $t \in$ $\operatorname{Spec}(\mathcal{A})$.

The centralizer $L$ of the image of $\phi$ in $C$ is a (closed) subgroup scheme of $C$, and $L$ is smooth over $\mathcal{B}$; cf. (3.6.1). Moreover, it follows from $(4.1 .1)(\mathrm{d})$ that $L_{t}$ is a Levi factor of $C_{t}$ for each $t \in \operatorname{Spec}(\mathcal{A})$, whence (b).

Since the subgroup scheme $L^{o}$ is reductive, one knows that $L$ - and hence $C$ - has a maximal torus by $(3.2 .1)$; this proves $(\mathrm{c})$.

According to $[J a 4, \S 5.3]$, one knows for each $t \in \operatorname{Spec}(\mathcal{A})$ that $N_{G}(X)_{\bar{t}}$ is the product of $C_{G}(X)_{\bar{t}}$ with the image of any cocharacter of $G_{\bar{t}}$ associated with $X$. Since the image of such a cocharacter centralizes some maximal torus in $C_{G}(X)_{\bar{t}}$, it follows that $\rho_{r, C}(t)+1=\rho_{r, N}(t)$. Using (5.7.1) it is now clear that $(d)$ is a consequence of $(c)$.

For later use, we observe that the proof of part (b) of the preceding Theorem actually proves the first assertion of the following:

(5.7.2). Assume that there is an $\mathcal{A}$-homomorphism $\phi: \mathbf{G}_{m} \rightarrow G$ such that the cocharacter $\phi_{t}$ of $G_{t}$ is associated to $X(t)$ for each $t \in \operatorname{Spec}(\mathcal{A})$.

(1) the centralizer of the image of $\phi$ in $C$ is a Levi factor $L$ of $C$.

(2) There is a smooth retraction $\rho: C \rightarrow L$ in the sense of (3.3.1); in particular, writing $R=\operatorname{ker} \rho$, there is an isomorphism of $\mathcal{A}$-group schemes $C \simeq L \ltimes R$.

Proof. We have observed that (1) was proved already. For the second assertion, write $\mathcal{A}[C]=\bigoplus_{n \in \mathbf{Z}} \mathcal{A}[C]_{n}$ as a direct sum of weight spaces for the action of $\mathbf{G}_{m}$ on $\mathcal{A}[C]$ given by Int* $\circ \phi$, where Int is the action of $C$ on itself by inner automorphisms.

Since $\phi_{t}$ is associated to $X(t)$, one knows by (4.1.1) that $\mathcal{A}[C]_{n, t}=0$ for any $t \in \operatorname{Spec}(\mathcal{A})$ whenever $n>0$. It follows that $\mathcal{A}[C]_{n}=0$ whenever $n>0$; i.e.

$$
\mathcal{A}[C]=\bigoplus_{n \leq 0} \mathcal{A}[C]_{n}
$$


Write $\mathcal{A}[C]_{<0}=\sum_{n<0} \mathcal{A}[C]_{n}$. Then $\mathcal{A}[L]=\mathcal{A}[C] / \mathcal{A}[C]_{<0}$, and the inclusion mapping $i: L \rightarrow C$ is given by the natural surjection $i^{*}: \mathcal{A}[C] \rightarrow$ $\mathcal{A}[C] / \mathcal{A}[C]_{<0}$. The Hopf algebra $\mathcal{A}[L]$ identifies naturally with $\mathcal{A}[C]_{0}$, and the inclusion map $\rho^{*}: \mathcal{A}[C]_{0} \rightarrow \mathcal{A}[C]$ defines a retraction $\rho: C \rightarrow L$. Since $\rho_{t}$ is evidently smooth for all $t \in \operatorname{Spec}(\mathcal{A})$, and since $C$ and $L$ are both flat over $\mathcal{A}$, [SGA 1, Exp. II, Cor. 2.2] shows that $\rho$ is a smooth mapping. In view of (3.3.1), this completes the proof of (2).

COROLlary. With assumptions as before, we have the following:

(a) Locally in the étale topology there are subgroup schemes $Q \subset L \subset G$ such that $L$ is a Levi subgroup scheme of a parabolic subgroup scheme of $G, Q$ is a a distinguished parabolic subgroup scheme of $L$, and $\left(L_{\bar{t}}, Q_{\bar{t}}\right)$ is the Bala-Carter datum of $X(t)$ for each $t \in \operatorname{Spec}(\mathcal{A})$.

(b) The root datum of the connected component of a Levi factor of $C_{G}(X)_{\bar{t}}$ is constant for $t \in \operatorname{Spec}(\mathcal{A})$.

Proof. For the proof of the corollary, we may replace $\mathcal{A}$ by a finite, étale, local extension; applying the Theorem for $G$, we may suppose that $C=C_{G}(X)$ has a maximal torus $T$. Now let $L=C_{G}(T)$; then $L$ is a Levi factor of a parabolic subgroup scheme of $G$, and $X \in \operatorname{Lie}(L)(\mathcal{A})$. Since $T$ is a maximal torus of $C_{L}(X)$, the Theorem applies also to $L$. Thus, we may suppose that there is an $\mathcal{A}$-homomorphism $\phi: \mathbf{G}_{m} \rightarrow L$ such that $\phi_{t}$ is a cocharacter of $L_{t}$ which is associated to $X(t)$ for each $t \in \operatorname{Spec}(\mathcal{A})$.

For (a), let $Q=P_{L}(\phi)$ be the parabolic subgroup scheme of $L$ determined by $\phi$. Since $\phi_{t}$ is associated with $X(t)$, one knows that $Q_{t}$ is the instability parabolic subgroup of $L_{t}$ determined by $X(t)$ (4.1.1). Since $T_{t}$ is a maximal torus of $C_{G}(X)_{t}$ for each $t \in \operatorname{Spec}(\mathcal{A})$, it is clear that $X(t)$ is distinguished in $\operatorname{Lie}(L)_{t}$. Thus indeed $\left(L_{t}, Q_{t}\right)$ is the Bala-Carter datum of $X(t)$.

For (b), note that $L_{t}$ is a Levi factor of $C_{G}(X)_{t}$ for each $t \in \operatorname{Spec}(\mathcal{A})$. So (b) follows from [SGA 3, III Exp. XXII Prop. 2.8].

\subsection{Proof of Theorem A}

Recall that $E$ is an algebraically closed field of characteristic 0, and that $F$ is an algebraically closed field of characteristic $p>0$. Theorem $\mathrm{A}$ is a consequence of the following more general result:

THEOREM. Let $G_{E}$ and $G_{F}$ be reductive groups respectively over $E$ and over $F$, assume that the root datum of $G_{E}$ coincides with that of $G_{F}$, 
and assume that $G_{F}$ is D-standard. Let $X_{E} \in \mathfrak{g}_{E}, X_{F} \in \mathfrak{g}_{F}$ be nilpotent elements with the same Bala-Carter data, let $C_{E}$ and $C_{F}$ be their respective centralizers, and let $L_{E} \subset C_{E}$ and $L_{F} \subset C_{F}$ be Levi factors (4.1.1). Then the root datum of $L_{E}^{o}$ may be identified with that of $L_{F}^{o}$.

Proof. Let $\mathcal{A}$ be the ring of Witt vectors ${ }^{6}$ [Se 79, II $\left.\S 6\right]$ with residue field an algebraic closure of the finite field $\mathbf{F}_{p}$. Using (4.4.2) we see that it is enough to prove Theorem A after replacing $F$ by the residue field of $\mathcal{A}$ and $E$ by an algebraic closure of the field of fractions of $\mathcal{A}$, and after replacing $X_{F}$ and $X_{E}$ by nilpotent elements with the given Bala-Carter datum.

Let $G$ be a split reductive group scheme over $\mathcal{A}$ with the same root datum as $G_{F}$ - for the existence, see e.g. [SGA 3, Exp. XXV, Thm. 1.1]. Then $G_{F}$ identifies with the closed fiber $G_{s}$ of $G$, and $G_{E}$ identifies with the generic fiber $G_{\bar{\eta}}$, where $\eta$ is the generic point of $\operatorname{Spec}(\mathcal{A})$.

Use Theorem 5.4 and the Bala-Carter theorem to find an equidimensional nilpotent section $X$ for which $X(s)$ is conjugate to $X_{F}$ and for which $X(\eta)$ is (geometrically) conjugate to $X_{E}$. We may and will replace $X_{F}$ by $X(s)$ and $X_{E}$ by $X(\eta)$.

If $C$ denotes the centralizer in $G$ of the nilpotent section $X$, it follows from part (b) of Corollary 5.7 that the root datum of a Levi factor of $C_{G}(X)_{\bar{t}}=C_{G_{\bar{t}}}(X(t))$ is constant for $t \in \operatorname{Spec}(\mathcal{A})$. This yields the desired result.

\section{§6. The group of components of a group scheme}

Again let $\mathcal{A}$ be a Noetherian, normal, local domain. Our goal in the section following this one is to investigate the groups $C_{t} / C_{t}^{o}$ where $t \in \operatorname{Spec}(\mathcal{A})$, where $C$ is the group scheme $C_{G}(X)$ for an equidimensional nilpotent section $X$, and where $G$ is assumed to be a $T$-standard reductive group scheme over $\mathcal{A}$. We first require some preliminaries, which we study in this section.

Let $H$ be a smooth and separated group scheme over $\mathcal{A}$. We are going to study the sheaf-quotient $H / H^{o}$, which we now describe.

\subsection{Sheaves}

If $X$ is an $\mathcal{A}$-scheme, an étale covering of $X$ is a family of étale $\mathcal{A}$ morphisms $\left(U_{i} \rightarrow X\right)_{i}$ of finite type, such that $X=\bigcup_{i} U_{i}$.

\footnotetext{
${ }^{6}$ The Witt vectors are just a convenient choice. In fact, one can use instead any normal, local Noetherian domain $\mathcal{A}$ with infinite residue field of characteristic $p>0$ whose field of fractions has characteristic 0 .
} 
Following [Mil 80, II $\S 1]$ we write $\operatorname{Spec}(\mathcal{A})_{\text {et }}$ for the (small) étale site of $\operatorname{Spec}(\mathcal{A})$. This means first of all that the underlying category of $\operatorname{Spec}(\mathcal{A})_{\text {et }}$ is the category $\operatorname{Et} / \operatorname{Spec}(\mathcal{A})$ of all schemes which are étale and of finite type over $\operatorname{Spec}(\mathcal{A})$; the morphisms of this category are just the morphisms of $\mathcal{A}$ schemes. Finally, $\operatorname{Spec}(\mathcal{A})_{\text {et }}$ is this category together with its (Grothendieck) topology defined by étale coverings of finite type.

A pre-sheaf of groups on $\operatorname{Spec}(\mathcal{A})_{\text {et }}$ is a contravariant functor

$$
\mathcal{F}: \operatorname{Et} / \operatorname{Spec}(\mathcal{A}) \longrightarrow \text { Groups; }
$$

the pre-sheaf $\mathcal{F}$ is a sheaf if the sequence $(\mathrm{S})$ of [Mil 80, II $\S 1$ p. 49] is exact for all coverings in $\mathrm{Et} / \operatorname{Spec}(\mathcal{A})$.

(6.1.1). [Mil 80, II Cor. 1.7 and Rem. 1.12] A group scheme $H$ over $\operatorname{Spec}(\mathcal{A})$ determines a sheaf of groups on $\operatorname{Spec}(\mathcal{A})_{\text {et }}$ by the rule $U \mapsto$ $\operatorname{Mor}_{\mathcal{A}}(H, U)$.

We say that a sheaf $\mathcal{F}$ on $\operatorname{Spec}(\mathcal{A})_{\text {et }}$ is representable if there is a group scheme $H$ such that $\mathcal{F}$ is isomorphic to the sheaf obtained from $H$ in (6.1.1). We sometimes abuse notation and write $H$ for the sheaf $\mathcal{F}$.

If $\mathcal{F}$ is a pre-sheaf on $\operatorname{Spec}(\mathcal{A})_{\text {et }}$ and if $x \in \operatorname{Spec}(\mathcal{A})$, the stalk $\mathcal{F}_{\bar{x}}$ is given by

$$
\mathcal{F}_{\bar{x}}=\lim _{\longrightarrow} \mathcal{F}(U)
$$

where the limit is taken over all étale neighborhoods $U$ of $x$ [Mil 80, II §2].

Let $\mathcal{A}_{\bar{x}}$ be the strict Henselization of $\mathcal{A}_{x}$ [Mil 80, I $\left.\S 4\right]$; thus $\mathcal{A}_{\bar{x}}$ is a Henselian local domain containing $\mathcal{A}_{x}$, and the residue field of the local ring $\mathcal{A}_{\bar{x}}$ is the separably closed field $k(\bar{x})$.

(6.1.2). [Mil 80, II Rem. 2.9(d)] Let $H$ be an $\mathcal{A}$-group scheme, and let $x \in \operatorname{Spec}(\mathcal{A})$. Then the stalk $H_{\bar{x}}$ of the sheaf $H$ identifies with the group of points $H\left(\mathcal{A}_{\bar{x}}\right)$.

Now let $J \subset H$ be a closed and normal subgroup scheme, and suppose that $H^{o} \subset J$. We write $H / J$ for the sheaf on $\operatorname{Spec}(\mathcal{A})_{\text {et }}$ obtained from the presheaf $U \mapsto H(U) / J(U)$.

(6.1.3). For each point $t \in \operatorname{Spec}(\mathcal{A})$, the stalk of the sheaf $H / J$ is given by

$$
(H / J)_{\bar{t}}=H(k(\bar{t})) / J(k(\bar{t})) .
$$


Proof. It follows from [Mil 80, II Thm. 2.11 and Thm. 2.15] that there is an exact sequence

$$
1 \longrightarrow J \longrightarrow H \longrightarrow H / J \longrightarrow 1
$$

of sheaves of groups on $\operatorname{Spec}(\mathcal{A})_{\text {et }}$. Since this sequence is exact on stalks, use (6.1.2) to see that

$$
(H / J)_{\bar{t}} \simeq H_{\bar{t}} / J_{\bar{t}} \simeq H\left(\mathcal{A}_{\bar{t}}\right) / J\left(\mathcal{A}_{\bar{t}}\right)
$$

So the assertion will follow once we see that $H\left(\mathcal{A}_{\bar{t}}\right) / J\left(\mathcal{A}_{\bar{t}}\right) \simeq H(k(\bar{t})) / J(k(\bar{t}))$. Since $H$ is smooth over $S$, the natural map $\phi: H\left(\mathcal{A}_{\bar{t}}\right) \rightarrow H(k(\bar{t})) / J(k(\bar{t}))$ is surjective (2.4.1). We have evidently $J\left(\mathcal{A}_{\bar{t}}\right) \subset \operatorname{ker} \phi$; it remains to see the reverse inclusion.

Recall that $H^{o}$ is open in $H$. Since $H^{o} \subset J$, it follows that $J \subset H$ is open as well. Since also $J \subset H$ is assumed to be closed, one sees that $J$ is a union of connected components of $H$. Let $g \in \operatorname{ker} \phi \subset H\left(\mathcal{A}_{\bar{t}}\right)$, and regard $g$ as a section $\operatorname{Spec}\left(\mathcal{A}_{\bar{t}}\right) \rightarrow H_{/ \mathcal{A}_{\bar{t}}}$. Since $g(\bar{t}) \in J_{\bar{t}}$ and since $\operatorname{Spec}\left(\mathcal{A}_{\bar{t}}\right)$ is connected, it follows that $g \in J\left(\mathcal{A}_{\bar{t}}\right)$. Thus ker $\phi=J\left(\mathcal{A}_{\bar{t}}\right)$ as required.

\subsection{Locally constant component groups}

Let $X$ be a scheme which is smooth and of finite type over $\mathcal{A}$. Write $s$ for the closed point of $\operatorname{Spec}(\mathcal{A})$.

(6.2.1). If $x \in X(k(\bar{s}))$, choose a finite separable extension $\ell \supset k$ such that $x \in X(\ell)$. Then there is a finite, étale, local extension $\mathcal{B} \supset \mathcal{A}$ and a section $y \in X(\mathcal{B})$ such that $\mathcal{B}$ has residue field $\ell$ and such that $x=y\left(s^{\prime}\right) \in$ $X(\ell)$, where $s^{\prime}$ denotes the closed point of $\operatorname{Spec}(\mathcal{B})$.

Proof. Since $X$ is of finite type, the existence of the required finite separable extension $\ell \supset k$ is immediate. As in [Mil 80, I Example 3.4] one may construct a finite, étale, local extension of $\mathcal{A}$ with residue field $\ell$; replacing $\mathcal{A}$ by this extension, we may as well suppose that $\ell=k$.

Now, using (2.4.1) we may find a section $y \in X\left(\mathcal{A}^{\mathrm{h}}\right)$ over the Henselization $\mathcal{A}^{\mathrm{h}}$ of $\mathcal{A}$ whose image in $X(k)$ is $x$. By construction [Mil 80, I $\S 4$ ] the Henselization $\mathcal{A}^{\mathrm{h}}$ is the limit of étale neighborhoods of $\mathcal{A}$; the existence of a suitable $\mathcal{B}$ follows at once.

Recall that $H$ is a group scheme which is smooth, separated, and of finite type over $\mathcal{A}$. 
(6.2.2). Let $J \subset H$ be a closed subgroup scheme, let $g \in H(\mathcal{A})$ be a section, and suppose that the image of $g$ in $H(K)$ lies in $J(K)$ where $K$ is the field of fractions of $\mathcal{A}$. Then $g \in J(\mathcal{A})$.

Proof. View $g$ is a morphism $\operatorname{Spec}(\mathcal{A}) \rightarrow H$. If $\eta$ denotes the generic point of $\operatorname{Spec}(\mathcal{A})$, the hypothesis means that the restriction of $g$ to the dense subset $\{\eta\}$ takes values in the closed subset $J$. Since $g$ is continuous, the image of $g$ must lie in $J$, as required.

(6.2.3). Let $J \subset H$ be a closed subgroup scheme. Then the natural map

$$
H(\mathcal{A}) / J(\mathcal{A}) \longrightarrow H(K) / J(K)
$$

is injective.

Proof. Suppose that $g \in H(\mathcal{A})$ and that $g J(\mathcal{A})$ is trivial in the group $H(K) / J(K)$. Then (the image of) $g$ determines an element of $J(K)$. Since $J$ is closed, it follows from $(6.2 .2)$ that $g \in J(\mathcal{A})$. This proves the required injectivity.

Proposition. Assume that $J$ is a closed subgroup scheme of $H$ containing $H^{o}$. Then

$$
\#(H / J)_{\bar{t}} \leq \#(H / J)_{\bar{\eta}} \quad \text { for each } t \in \operatorname{Spec}(\mathcal{A})
$$

where $\eta$ is the generic point of $\operatorname{Spec}(\mathcal{A})$. Moreover, the following are equivalent:

(i) The sheaf $H / J$ on $\operatorname{Spec}(\mathcal{A})_{\text {et }}$ is represented by a finite, étale $\mathcal{A}$-group scheme.

(ii) The sheaf $H / J$ on $\operatorname{Spec}(\mathcal{A})_{\text {et }}$ is locally constant.

(iii) $\#(H / J)_{\bar{t}}$ is constant on $\operatorname{Spec}(\mathcal{A})$.

Proof. It suffices to prove the inequality $\#(H / J)_{\bar{t}} \leq \#(H / J)_{\bar{\eta}}$ in case $t$ is the closed point $s \in \operatorname{Spec}(\mathcal{A})$. Indeed, if $t \in \operatorname{Spec}(\mathcal{A})$ is arbitrary, one replaces $\mathcal{A}$ by the normal local ring $\mathcal{A}_{t}$; since $t$ is the closed point of $\operatorname{Spec}\left(\mathcal{A}_{t}\right)$ one then deduces the required inequality.

In view of $(6.1 .3)$, we have $(H / J)_{\bar{s}}=H(k(\bar{s})) / J(k(\bar{s}))$; choose a finite separable extension $\ell \supset k=k(s)$ and elements $x_{1}, \ldots, x_{n} \in H(\ell)$ such that the cosets of the $x_{i}$ are precisely the elements of $H(k(\bar{s})) / J(k(\bar{s}))$. 
We may now use (6.2.1) to find a finite, étale, local extension $\mathcal{B} \supset \mathcal{A}$ with residue field $\ell$, and sections $y_{1}, \ldots, y_{n} \in H(\mathcal{B})$ such that $y_{i}\left(s^{\prime}\right)=x_{i}$ in $H(\ell)$ for $1 \leq i \leq n$, where $s^{\prime} \in \operatorname{Spec}(\mathcal{B})$ is the point lying over $s$.

Since $J$ is closed in $H$, it follows from (6.2.3) that the classes in $H(k(\bar{\eta})) / J(k(\bar{\eta}))$ of the elements $x_{i}(\eta) \in H(k(\bar{\eta}))$ are all distinct. Thus indeed $\#(H / J)_{\bar{\eta}} \geq n$ as required.

We now prove that (i) implies (iii); we suppose that there is a finite étale group scheme $\Gamma$ representing the sheaf $H / J$. Since $\mathcal{A}_{\bar{x}}$ is Henselian, application of [Mil 80, I Theorem 4.2(c)] shows that the coordinate ring of the finite étale $\mathcal{A}_{\bar{x}}$-group scheme $\Gamma_{/ \mathcal{A}_{\bar{x}}}$ is a direct product of local rings each with residue field $k(\bar{x})$. It follows at once that $\# \Gamma_{\bar{\eta}}=\# \Gamma_{\bar{x}}$ where $\eta$ is the generic point of $\operatorname{Spec}(\mathcal{A})$, so that indeed $\#(H / J)_{\bar{x}}$ is constant on $\operatorname{Spec}(\mathcal{A})$.

We next prove that (iii) implies (ii). Write $n$ for the constant value of $\#(H / J)_{\bar{x}}$. To prove (ii), we must show that $H / J$ is locally constant. It is enough to prove that $H / J$ is constant after we replace $\mathcal{A}$ by a finite, étale, local extension; thus, we may arrange that there are sections $y_{1}, \ldots, y_{n} \in H(\mathcal{A})$ for which the cosets $y_{i}(s) J(k(s))$ are the $n$ distinct elements of $H(k(s)) / J(k(s))=(H / J)_{\bar{s}}$. It follows from (6.2.3) that the cosets $y_{i}(\eta) J(K)$ are all distinct, where $K=k(\eta)$ is the field of fractions of $\mathcal{A}$.

Suppose now that $\mathcal{B} \supset \mathcal{A}$ is any finite, étale, local extension and that $z \in H(\mathcal{B})$ is any section. Since by assumption $(H / J)_{\bar{\eta}}$ has $n$ points, we may find $1 \leq i \leq n$ such that $z y_{i}^{-1} \in J(L)$ where $L$ is the field of fractions of $\mathcal{B}$. But $z y_{i}^{-1} \in H(\mathcal{B})$; it then follows from (6.2.3) that $z y_{i}^{-1} \in J(\mathcal{B})$. Thus the $y_{i}$ are a full set of coset representatives for the quotient $H(\mathcal{B}) / J(\mathcal{B})$.

If now $t \in \operatorname{Spec}(\mathcal{A})$ is arbitrary, one knows by (6.2.1) that there is some finite, étale, local extension $\mathcal{B} \supset \mathcal{A}$ such that $H(\mathcal{B}) / J(\mathcal{B}) \rightarrow(H / J)_{\bar{t}}$ is surjective. Since the group $(H / J)_{\bar{t}}$ has exactly $n$ elements, the above argument shows the natural mapping $H(\mathcal{A}) / J(\mathcal{A}) \rightarrow(H / J)_{\bar{t}}$ to be an isomorphism. It follows that $H / J$ is a constant sheaf on $\operatorname{Spec}(\mathcal{A})_{\text {et }}$, as required.

The fact that (ii) implies (i) follows from [Mil 80, V Prop. 1.1] ${ }^{7}$.

\section{3. Étale central isogenies of group schemes}

Let $H$ and $H_{1}$ be separated group schemes which are smooth and of finite type over $\mathcal{A}$ for which $H^{o}$ and $H_{1}^{o}$ are reductive. Assume that $f$ : $H_{1} \rightarrow H$ is an étale central isogeny over $\mathcal{A}$; this condition means that the

\footnotetext{
${ }^{7}$ Note that [Mil 80, Chapter V Prop. 1.1] is stated for sheaves of Abelian groups, but commutativity is not used in the proof.
} 
$\mathcal{A}$-morphism $f$ is étale, finite, and faithfully flat, and that $\operatorname{ker} f$ is central in $H$.

(6.3.1). Write $f^{o}=f_{\mid H_{1}^{o}}: H_{1}^{o} \rightarrow H^{o}$ for the restriction of $f$ to $H_{1}^{o}$. Then $f^{o}$ is also an étale central isogeny.

Proof. Since ker $f$ is central in $H_{1}$, it is clear that ker $f^{o}$ is central in $H_{1}^{o}$. Now, $H_{1}^{o}$ and $H^{o}$ are both smooth over $\mathcal{A}$. Since the $k(t)$-morphism $f_{t}:\left(H_{1}\right)_{t} \rightarrow H_{t}$ is étale and surjective for each $t \in \operatorname{Spec}(\mathcal{A})$, it is clear that the same holds for $f_{t}^{o}:\left(H_{1}^{o}\right)_{t} \rightarrow H_{t}^{o}$; thus $f^{o}$ is étale and surjective; in particular, $f^{o}$ is faithfully flat.

It remains only to show that $f^{o}$ is finite. Since $H_{1}^{o}$ is reductive, we know that $H_{1}^{o} \rightarrow H_{1}$ is also a closed immersion (3.4.2), hence finite. Thus the composition of finite morphisms

$$
H_{1}^{o} \longrightarrow H_{1} \stackrel{f}{\longrightarrow} H
$$

is itself finite; it is then immediate that $f^{o}$ is finite as well.

(6.3.2). The étale sheaf $H / H^{o}$ is represented by a finite étale group scheme if and only if that is so for $H_{1} / H_{1}^{o}$.

Proof. One knows that ker $f$ identifies with the fiber product

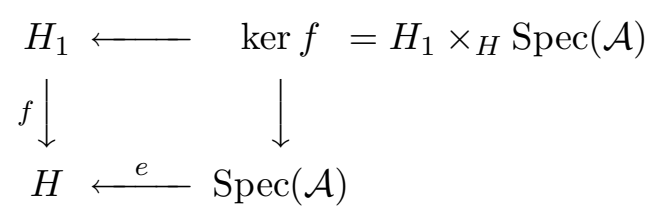

where $e$ is the identity section of $H$. Since $f$ is an étale isogeny, it follows that the subgroup scheme ker $f \subset H_{1}$ is finite and étale over $\operatorname{Spec}(\mathcal{A})$.

In view of (6.3.1), the same argument shows that the subgroup scheme ker $f^{o} \subset H_{1}^{o}$ is finite and étale over $\operatorname{Spec}(\mathcal{A})$ as well.

Since the sheaves ker $f$ and $\operatorname{ker} f^{o}$ are represented by a finite étale group schemes, one knows by Proposition 6.2 that \# $(\operatorname{ker} f)_{\bar{t}}$ is constant and $\#\left(\operatorname{ker} f^{o}\right)_{\bar{t}}$ is constant for $t \in \operatorname{Spec}(\mathcal{A})$. Thus the quotient sheaf $(\operatorname{ker} f) /\left(\operatorname{ker} f^{o}\right)$ has the property that $\#\left((\operatorname{ker} f) /\left(\operatorname{ker} f^{o}\right)\right)_{\bar{t}}$ is constant for $t \in \operatorname{Spec}(\mathcal{A})$.

The sequence of sheaves on $\operatorname{Spec}(\mathcal{A})_{\text {et }}$

$$
1 \longrightarrow(\operatorname{ker} f) /\left(\operatorname{ker} f^{o}\right) \longrightarrow H_{1} / H_{1}^{o} \longrightarrow H / H^{o} \longrightarrow 1
$$


is exact, since it is exact on the stalks of each geometric point $\bar{t}$ for $t \in$ $\operatorname{Spec}(\mathcal{A})$. It follows at once that $\#\left(H_{1} / H_{1}^{o}\right)_{\bar{t}}$ is constant on $\operatorname{Spec}(\mathcal{A})$ if and only if $\#\left(H / H^{o}\right)_{\bar{t}}$ is constant on $\operatorname{Spec}(\mathcal{A})$. The result now holds by Proposition 6.2.

\subsection{The centralizer of a diagonalizable subgroup scheme}

Let $H$ be as in the previous section; thus $H$ is smooth and of finite type over $\mathcal{A}$, and $H^{o}$ is reductive.

We first recall the following result regarding the Weyl group of a maximal torus of a (connected and) reductive group scheme:

(6.4.1). [SGA 3, Exp. XIX Thm. 2.5] Suppose that $H=H^{o}$ is reductive with connected geometric fibers, let $T \subset H$ be a maximal torus, and let $N_{H}(T)$ be the normalizer in $H$ of $T$. Then the quotient $W=N_{H}(T) / T=$ $N_{H}(T) / C_{H}(T)$ is represented by a finite and étale group scheme over $\mathcal{A}$. In particular, $N_{H}(T) / T$ is a locally constant sheaf on $\operatorname{Spec}(\mathcal{A})_{\text {et }}$.

Suppose now that $D \subset H$ is a closed and smooth ${ }^{8}$ subgroup of multiplicative type which is contained in a maximal torus $T \subset H$, and let $L=C_{H}(D)$ be the centralizer in $H$ of $D$; recall (3.6.2) that $L^{o}$ is reductive.

(6.4.2). Assume that $H=H^{o}$. Then the sheaf $L / L^{o}$ is represented on

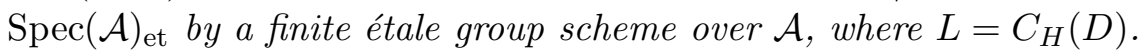

Proof. Since $L^{o}$ is reductive, one knows by (3.4.2) that $L^{o}$ is closed in $L$. If $\eta \in \operatorname{Spec}(\mathcal{A})$ is the generic point, then we have

$$
(*) \quad \#\left(L / L^{o}\right)_{\bar{\eta}} \geq \#\left(L / L^{o}\right)_{\bar{t}}
$$

for each $t \in \operatorname{Spec}(\mathcal{A})$ by the first assertion of Proposition 6.2. If we show that equality holds in $(*)$ for each $t \in \operatorname{Spec}(\mathcal{A})$, then the desired result follows from the equivalence of (i) and (iii) of that same Proposition.

Recall that $T \subset L^{o}$ is a maximal torus centralized by $D$. Since all maximal tori of $L^{o}$ are conjugate on the geometric fibers $L^{o}{ }_{\bar{t}}$ for $t \in \operatorname{Spec}(\mathcal{A})$, the natural map

$$
N_{L}(T) / N_{L^{o}}(T) \longrightarrow L / L^{o}
$$

\footnotetext{
${ }^{8}$ The assumption that $D$ is smooth is imposed here only for lack of adequate reference that $L^{o}=C_{H}(D)^{o}$ is reductive; see the remark following (3.6.2). Given that $L^{o}$ is reductive, the proofs of (6.4.2) and (6.4.3) are independent of the smoothness of $D$.
} 
determines an isomorphism on each geometric fiber and thus defines an isomorphism of sheaves on $\operatorname{Spec}(\mathcal{A})_{\mathrm{et}}$.

After replacing $\mathcal{A}$ by a finite, étale, local extension, the characterization (6.4.1) shows that we may suppose $N_{H}(T) / T$ to be a constant sheaf. If $n$ denotes the (constant) order of the geometric stalks, we may choose sections $x_{1}, x_{2}, \ldots, x_{n} \in N_{H}(T)(\mathcal{A})$ such that $\left(N_{H}(T) / T\right)_{\bar{t}}=\left\{\overline{x_{1}(t)}, \ldots, \overline{x_{n}(t)}\right\}$ for each $t \in \operatorname{Spec}(\mathcal{A})$, where $\overline{x_{i}(t)}$ denotes the coset $x_{i}(t) T(k(\bar{t}))$.

Now let $y \in N_{L}(T)(k(\eta))$. Regarding $y$ as an element of $N_{H}(T)(k(\eta))$, we may find $1 \leq i \leq n$ such that $x_{i}(\eta) y^{-1}=z \in T(K)$.

Since the torus $T$ contains $D$, the element $z$ centralizes $D_{\eta}$; it follows that also $x_{i}(\eta)=y z$ centralizes $D$-i.e. $x_{i}(\eta) \in N_{L}(T)(k(\eta))$. It now follows from $(6.2 .2)$ that $x_{i} \in N_{L}(T)(\mathcal{A})$.

We have now proved that the natural map

$$
N_{L}(T)(\mathcal{A}) / N_{L^{o}}(T)(\mathcal{A}) \longrightarrow N_{L}(T)(k(\eta)) / N_{L^{o}}(T)(k(\eta))
$$

is surjective. Since for any $t \in \operatorname{Spec}(\mathcal{A})$ the natural map

$$
N_{L}(T)(\mathcal{A}) / N_{L^{o}}(T)(\mathcal{A}) \longrightarrow\left(N_{L}(T) / N_{L^{o}}(T)\right)_{\bar{t}}
$$

is injective by the definition of $L^{o}$, it follows that equality holds in $(*)$, as required.

We will now prove that the assertion of (6.4.2) remains true without the assumption that $H=H^{o}$.

(6.4.3). If the étale sheaf $H / H^{o}$ is represented by a finite étale group scheme over $\mathcal{A}$, then $L / L^{o}$ is represented by a finite étale group scheme over $\mathcal{A}$ as well.

Proof. Consider the subgroup $L_{1}=C_{H^{\circ}}(D)$; we have

$$
L^{o} \subset L_{1} \subset L \text {. }
$$

Thus there is an exact sequence of sheaves on $\operatorname{Spec}(\mathcal{A})_{\text {et }}$

$$
1 \longrightarrow L_{1} / L^{o} \longrightarrow L / L^{o} \longrightarrow L / L_{1} \longrightarrow 1 \text {. }
$$

It follows from (6.4.2) that $L_{1} / L^{o}$ is represented by a finite étale group scheme over $\mathcal{A}$. 
Suppose we show that the order of the geometric fiber $\left(L / L_{1}\right)_{\bar{t}}$ is independent of $t \in \operatorname{Spec}(\mathcal{A})$. Using the exactness of the above sequence of groups, we see that the order of the geometric fiber $\left(L / L^{o}\right)_{\bar{t}}$ is independent of $t \in \operatorname{Spec}(\mathcal{A})$. Since $L^{o}$ is reductive and hence closed in $L$ by (3.4.2), it follows from Proposition 6.2 that $L / L^{o}$ is locally constant and represented by a finite étale group scheme over $\mathcal{A}$, as required.

It now remains to prove that $\#\left(L / L_{1}\right)_{\bar{t}}$ is constant. Since $L_{1}=C_{H^{o}}(D)$ is closed in $H^{o}$ and since $H^{o}$ is closed in $H$ by (3.4.2), we have that $L_{1}$ is closed in $H$. Since $L=C_{H}(D)$ is closed in $H, L_{1}$ is closed in $L$ as well. Thus we may apply Proposition 6.2 to study the quotient $L / L_{1}$. That Proposition shows especially that

$$
(*) \quad \#\left(L / L_{1}\right)_{\bar{\eta}} \geq \#\left(L / L_{1}\right)_{\bar{t}}
$$

for each $t \in \operatorname{Spec}(\mathcal{A})$, and the desired result holds if we prove that equality holds in $(*)$ for each $t$.

Recall that $T$ is a maximal torus of $H^{o}$ containing $D$. Arguing as in (6.4.2), we see that the natural map $N_{H}(T) / N_{H^{o}}(T) \rightarrow H / H^{o}$ is an isomorphism of sheaves on $\operatorname{Spec}(\mathcal{A})_{\text {et }}$. Since $T \subset L_{1}$, a similar argument shows that the natural map $N_{L}(T) / N_{L_{1}}(T) \rightarrow L / L_{1}$ is an isomorphism of sheaves on $\operatorname{Spec}(\mathcal{A})_{\text {et}}$.

Recall that we have assumed $H / H^{o}$ to be represented by a finite étale group scheme; thus $N_{H}(T) / N_{H^{o}}(T) \simeq H / H^{o}$ is locally constant. Since $H^{o}$ is reductive, also $N_{H^{o}}(T) / T$ is locally constant by (6.4.1). Thus after replacing $\mathcal{A}$ by a finite, étale, local extension, we may suppose that $N_{H}(T) / N_{H^{o}}(T)$ and $\left.N_{H^{o}}(T) / T\right)$ are constant sheaves on $\operatorname{Spec}(\mathcal{A})_{\text {et }}$. Choose a complete set of representatives

$$
x_{1}, \ldots, x_{n} \in N_{H}(T)(\mathcal{A}) \text { for the elements of } \quad N_{H}(T)(\mathcal{A}) / N_{H^{o}}(T)(\mathcal{A}),
$$

and a complete set of representatives

$$
y_{1}, \ldots, y_{m} \in N_{H^{o}}(T)(\mathcal{A}) \quad \text { for the elements of } \quad N_{H^{o}}(T)(\mathcal{A}) / T(\mathcal{A}) .
$$

If now $w \in N_{L}(T)(k(\eta))$, we have $x_{i}(\eta) y_{j}(\eta) w^{-1}=z \in T(k(\eta))$ for some $1 \leq i \leq n$ and $1 \leq j \leq m$. But then $x_{i}(\eta) y_{j}(\eta)=z y$ centralizes $D_{\eta}$, hence $x_{i}(\eta) y_{j}(\eta) \in N_{L}(T)(k(\eta))$. Since $x_{i} y_{j} \in N_{H}(T)(\mathcal{A})$, it follows from (6.2.2) that $x_{i} y_{j} \in N_{L}(T)(\mathcal{A})$.

This shows the natural map

$$
N_{L}(T)(\mathcal{A}) / N_{L_{1}}(T)(\mathcal{A}) \longrightarrow N_{L}(T)(k(\eta)) / N_{L_{1}}(T)(k(\eta))
$$


to be surjective.

Notice that the natural map $\left(L / L_{1}\right)_{\bar{t}} \rightarrow\left(H / H^{o}\right)_{\bar{t}}$ is injective for each $t \in \operatorname{Spec}(\mathcal{A})$, hence $L / L_{1} \rightarrow H / H^{o}$ is an injective mapping of sheaves on $\operatorname{Spec}(\mathcal{A})_{\text {et }}$. Since the natural map $H(\mathcal{A}) / H^{o}(\mathcal{A}) \rightarrow\left(H / H^{o}\right)_{\bar{t}}$ is injective for each $t \in \operatorname{Spec}(\mathcal{A})$ by the definition of $H^{o}$, it follows that the natural map $L(\mathcal{A}) / L_{1}(\mathcal{A}) \rightarrow\left(L / L_{1}\right)_{\bar{t}}$ is injective. Thus indeed equality holds in $(*)$, as required.

\section{$\S 7$. The component group of a nilpotent centralizer}

Let $\mathcal{A}$ be a local, normal, Noetherian domain, let $G$ be a $T$-standard reductive group scheme over $\mathcal{A}$. Fix throughout this section an equidimensional nilpotent section

$$
X \in \operatorname{Lie}(G)(\mathcal{A})=\mathfrak{g}(\mathcal{A})
$$

Let $C=C_{G}(X)$ be the centralizer in $G$ of $X$, and recall that $C$ is a smooth group scheme over $\mathcal{A}$; see Proposition 5.2.

\subsection{Replacing $C / C^{o}$ by $L / L^{o}$}

According to Theorem 5.7, $C$ has a Levi factor locally in the étale topology. Thus after replacing $\mathcal{A}$ by a finite étale local extension, we may assume that there is a Levi factor $L \subset C$. In this situation, we have the following:

(7.1.1). There is an isomorphism $C / C^{o} \simeq L / L^{o}$ of sheaves on $\operatorname{Spec}(\mathcal{A})_{\text {et }}$.

Proof. It follows from [MS 03, Prop. 12] that the natural map defines an isomorphism

$$
\left(L / L^{o}\right)_{\bar{t}} \simeq\left(C / C^{o}\right)_{\bar{t}} \text { for each } t \in \operatorname{Spec}(\mathcal{A}) ;
$$

this means that the natural sheaf map $L / L^{o} \rightarrow C / C^{o}$ is an isomorphism on stalks and is thus an isomorphism.

\subsection{The adjoint case}

Assume that $G$ is a semisimple group scheme over $\mathcal{A}$, and that $G$ is of adjoint type - i.e. that $G_{t}$ is adjoint for each $t \in \operatorname{Spec}(\mathcal{A})$; cf. Section 3.2.

TheOREM. [MS 03] The quotient $C / C^{o}$ is represented on $\operatorname{Spec}(\mathcal{A})_{\mathrm{et}}$ by a finite étale group scheme over $\mathcal{A}$. In particular, $\left(C / C^{o}\right)_{\bar{t}} \simeq\left(C / C^{o}\right)_{\overline{t^{\prime}}}$ are all $t, t^{\prime} \in \operatorname{Spec}(\mathcal{A})$. 
Proof. Replacing $\mathcal{A}$ by a finite, étale, local extension we may suppose that $C$ has a Levi factor $L$. According to (7.1.1), we know that $C / C^{o} \simeq$ $L / L^{o}$. Since $L^{o}$ is reductive, $L^{o}$ is closed in $L$ (3.4.2). Thus we may apply Proposition 6.2. According to that Proposition, the Theorem will follow once we know that $\left|\left(L / L^{o}\right)_{\bar{t}}\right|$ is constant for $t \in \operatorname{Spec}(\mathcal{A})$. Since for each $t \in$ $\operatorname{Spec}(\mathcal{A})$, we have assumed $G_{\bar{t}}$ to be semisimple and adjoint, that constancy follows from [MS 03, Theorem 36].

\section{3. Étale central isogenies}

Let $G$ and $G_{1}$ be $T$-standard reductive group schemes over $\mathcal{A}$, and let $f: G \rightarrow G_{1}$ be an étale central isogeny. This means that $f$ is finite, étale, and faithfully flat, and $\operatorname{ker} f$ is central in $G$.

(7.3.1). df : $\operatorname{Lie}(G)(\mathcal{A}) \rightarrow \operatorname{Lie}\left(G_{1}\right)(\mathcal{A})$ is an isomorphism of $\mathcal{A}$-modules.

Proof. Since $f_{t}: G_{t} \rightarrow G_{1, t}$ is a separable central isogeny for each $t \in \operatorname{Spec}(\mathcal{A})$, one knows that $\operatorname{dim} G_{t}=\operatorname{dim} G_{1, t} . \quad \operatorname{Thus}, \operatorname{Lie}(G)(\mathcal{A})$ and $\operatorname{Lie}\left(G_{1}\right)(\mathcal{A})$ are free $\mathcal{A}$-modules of the same rank. Writing $\mathfrak{m}$ for the unique maximal ideal of $\mathcal{A}$, one knows that

$$
\operatorname{Lie}(G)(k(s))=\operatorname{Lie}(G)(\mathcal{A}) / \mathfrak{m} \operatorname{Lie}(G)(\mathcal{A}),
$$

with a similar statement for $G_{1}$. Since $d f_{s}: \operatorname{Lie}(G)_{s} \rightarrow \operatorname{Lie}\left(G_{1}\right)_{s}$ is an isomorphism, it follows from the Nakayama lemma that $d f: \operatorname{Lie}(G)(\mathcal{A}) \rightarrow$ $\operatorname{Lie}\left(G_{1}\right)(\mathcal{A})$ is an isomorphism.

Let $X \in \operatorname{Lie}(G)(\mathcal{A})$ and $X_{1} \in \operatorname{Lie}\left(G_{1}\right)(\mathcal{A})$ be nilpotent sections, and suppose that $d f(X)=d f\left(X_{1}\right)$. Write $C=C_{G}(X), N=N_{G}(X), C_{1}=$ $C_{G_{1}}\left(X_{1}\right)$ and $N_{1}=N_{G_{1}}\left(X_{1}\right)$.

(7.3.2). $X$ is an equidimensional nilpotent section of $\operatorname{Lie}(G)$ if and only if $X_{1}$ is an equidimensional nilpotent section of $\operatorname{Lie}\left(G_{1}\right)$.

Proof. Indeed, it is clear for each $t$ that $f_{t}$ restricts to a separable isogeny

$$
f_{t \mid C_{t}}: C_{t} \longrightarrow C_{1, t}
$$

of $k(t)$-group schemes, whence $\operatorname{dim} C_{t}=\operatorname{dim} C_{1, t}$.

(7.3.3). Locally in the étale topology there are Levi factors $L \subset C$ and $L_{1} \subset C_{1}$ for which $f_{\mid L}$ determines a finite, étale, and faithfully flat map of group schemes $f_{\mid L}: L \rightarrow L_{1}$. 
Proof. After possibly replacing $\mathcal{A}$ by a finite, étale, local extension, we may use Theorem 5.7(a) to find a homomorphism $\phi: \mathbf{G}_{m} \rightarrow G$ such that $\phi_{t}$ is a cocharacter of $G_{t}$ associated to $X(t)$ for each $t \in \operatorname{Spec}(\mathcal{A})$. If $\psi=f \circ \phi$, it follows from [Mc 04, Lem. 14] that $\psi_{t}$ is a cocharacter of $G_{1, t}$ associated with $X_{1}(t)$ for each $t$.

Using (5.7.2), one knows that the centralizer $L$ of the image of $\phi$ in $C$ is a Levi factor, and the centralizer $L_{1}$ of the image of $\psi$ in $C_{1}$ is a Levi factor.

It is clear that $f$ restricts to a morphism $f_{\mid L}: L \rightarrow L_{1}$; we only must argue that $f_{\mid L}$ is finite, étale, and faithfully flat.

For that, we notice first that $f_{t \mid L_{t}}: L_{t} \rightarrow L_{1, t}$ is a separable $k(t)$-isogeny for each $t \in \operatorname{Spec}(\mathcal{A})$. It follows at once that $f$ is faithfully flat. Moreover, since $L$ and $L_{1}$ are smooth over $\mathcal{A}$, [SGA 1, Exp. II, Cor. 2.2] shows that $f_{\mid L}$ is smooth. Since $f$ is finite, it follows that $f_{\mid L}$ is quasi-finite. But then $f_{\mid L}$ is étale [SGA 1, Exp. II, Cor. 1.4].

It remains to show that $f_{\mid L}$ is a finite morphism. Note first that the inclusions $C \subset G$ and $C_{1} \subset G_{1}$ are closed embeddings (see Section 2.3), and the inclusions $L \subset C$ and $L_{1} \subset C_{1}$ are closed embeddings (3.6.1). Since $f$ is finite hence proper [Mil 80, I Prop. 1.4], the composition $L \subset C \subset G \stackrel{f}{\rightarrow} G_{1}$ is a proper map. Since $L_{1} \subset G_{1}$ is a closed embedding, it follows from [Li 02, Prop. 3.3.16] that $f_{\mid L}: L \rightarrow L_{1}$ is proper. Since $f_{\mid L}$ is quasifinite and proper, $f_{\mid L}$ is finite by [Mil 80, I Cor. 1.1].

Proposition. The sheaf $C / C^{o}$ is represented on $\operatorname{Spec}(\mathcal{A})_{\mathrm{et}}$ by a finite étale $\mathcal{A}$-scheme if and only if that is so for $C_{1} / C_{1}^{o}$.

Proof. Replacing $\mathcal{A}$ be a finite, étale local extension, we may use (7.3.3) to find Levi factors $L \subset C$ and $L_{1} \subset C_{1}$ for which $f_{\mid L}$ determines a finite, étale, faithfully flat morphism $f: L \rightarrow L_{1}$. Then we have

$$
C / C^{o} \simeq L / L^{o} \quad \text { and } \quad C_{1} / C_{1}^{o} \simeq L_{1} / L_{1}^{o}
$$

by (7.1.1). Since $L^{o}$ and $L_{1}^{o}$ are reductive, the Proposition now follows by applying (6.3.2).

\subsection{The centralizer of a torus}

Let $S \subset G$ be a torus, and let $M=C_{G}(S)$ be the centralizer of $S$ in $G$. Then $M$ is a reductive group scheme over $\mathcal{A}$ with connected geometric fibers. Suppose that $X \in \operatorname{Lie}(M)(\mathcal{A})$ is an equidimensional nilpotent section, and write $C=C_{G}(X)$ and $C_{M}=C_{M}(X)$. 
(7.4.1). $X$ is an equidimensional nilpotent section of $\operatorname{Lie}(G)(\mathcal{A})$ as well. In particular, $C$ is equidimensional and hence smooth.

Proof. It follows from Corollary 5.7 that there are subgroup schemes $Q \subset L \subset M$ such that $L$ is a Levi subgroup scheme of a parabolic subgroup scheme of $M, Q$ is a parabolic subgroup scheme of $M$, and $\left(L_{\bar{t}}, Q_{\bar{t}}\right)$ is the Bala-Carter datum of $X(t)$ for each $t \in \operatorname{Spec}(\mathcal{A})$.

Now use Theorem 5.4 to find an equidimensional nilpotent section $Y \in \operatorname{Lie}(G)(\mathcal{A})$ whose Bala-Carter datum coincides with that of $X(\eta)$. Since the Bala-Carter datum of $X(t)$ in $\operatorname{Lie}(G)_{t}$ is determined by the Bala-Carter datum of $X(t)$ in $\operatorname{Lie}(M)_{t}$, it follows from the Bala-Carter theorem that $X(t)$ and $Y(t)$ are conjugate by an element of $G(k(\bar{t}))$. Since $Y$ is equidimensional, it follows that $X$ is equidimensional as well.

Proposition. If the sheaf $C / C^{o}$ is represented on $\operatorname{Spec}(\mathcal{A})_{\text {et }}$ by a finite étale $\mathcal{A}$-group scheme, the same holds for $C_{M} / C_{M}^{o}$.

Proof. In view of (7.4.1), one knows that $C$ and $C_{M}$ are smooth over $\mathcal{A}$. Thus the centralizers $L \subset C$ and $L_{1} \subset C_{M}$ of the image of $\phi$ are closed subgroup schemes which are smooth over $\mathcal{A}$. Using (5.7.2), one knows that $L$ is a Levi factor in $C$ and that $L_{1}$ is a Levi factor in $C_{M}$. Now,

$$
C / C^{o} \simeq L / L^{o} \quad \text { and } \quad C_{M} / C_{M}^{o} \simeq L_{1} / L_{1}^{o}
$$

by (7.1.1).

Since $\phi$ evidently centralizes the torus $S$, it is clear that $S \subset L_{1}^{o} \subset L^{o}$. Since the centralizer of $S$ in $L^{o}$ is a reductive subgroup scheme, we may find a maximal torus $T \subset L^{o}$ containing $S$ - use (3.2.1) and (3.6.3). The Proposition now follows from (6.4.3).

Remark. It is not clear - to the author, at least - whether the Proposition is true when $T$ is replaced by any diagonalizable subgroup scheme $D \subset G$; in the notation of the (proof of the) Proposition, the difficulty lies in the fact that $D$ need not be contained in a maximal torus of $L_{1}^{o}$, so that (6.4.3) is inadequate.

\subsection{The component group of $C$}

Let $G$ be a $T$-standard reductive group scheme over $\mathcal{A}$. Let $X \in \mathfrak{g}(\mathcal{A})$ be an equidimensional nilpotent section, let $C=C_{G}(X)$, and assume that the pair $(G, X)$ is allowable. 
Theorem. The étale sheaf $C / C^{o}$ is represented on $\operatorname{Spec}(\mathcal{A})_{\text {et }}$ by a finite étale group scheme over $\mathcal{A}$. In particular, $\left(C / C^{o}\right)_{\bar{t}} \simeq\left(C / C^{o}\right)_{\bar{t}^{\prime}}$ for all $t, t^{\prime} \in \operatorname{Spec}(\mathcal{A})$.

Proof. Let first $G$ be semisimple and assume the fiber characteristics are all very good for $G$. After replacing $\mathcal{A}$ by a finite, étale, local extension, we may suppose that $G$ is split; let $G_{\mathrm{ad}}$ be the corresponding group of adjoint type and let $f: G \rightarrow G_{\text {ad }}$ be the corresponding map (3.2.3). In view of our assumptions, (3.8.1) shows that $f$ is an étale central isogeny. Since the assertion of the Theorem holds for the pair $G_{\mathrm{ad}}, d f(X)$ by Theorem 7.2, the assertion for $G$ now follows from Proposition 7.3.

It is then clear that the assertion of the Theorem holds when $G$ is a group of the form $H=H_{1} \times S$ where $S$ is a torus and where $H_{1}$ is semisimple and the characteristic of $k(t)$ is very good for $H_{t}$ for each $t \in \operatorname{Spec}(\mathcal{A})$. If $S_{0}$ is a torus in the group $H$, Proposition 7.4 shows that the Theorem holds for $M=C_{H}\left(S_{0}\right)$.

If $G$ is any $T$-standard group, there is an étale isogeny between $G$ and a group of the form $M$ as above; thus the assertion of the Theorem follows from another application of Proposition 7.3.

\subsection{Proof of Theorem B}

Recall that $E$ is an algebraically closed field of characteristic 0 , and that $F$ is an algebraically closed field of characteristic $p>0$. Theorem $\mathrm{B}$ is a consequence of the following more general result:

THEOREM. Let $G_{E}$ and $G_{F}$ be reductive groups respectively over $E$ and over $F$, assume that the root datum of $G_{E}$ coincides with that of $G_{F}$, and assume that $G_{F}$ is T-standard. Let $X_{E} \in \mathfrak{g}_{E}, X_{F} \in \mathfrak{g}_{F}$ be nilpotent elements with the same Bala-Carter data, and let $C_{E}$ and $C_{F}$ be their respective centralizers. Then $C_{E} / C_{E}^{o} \simeq C_{F} / C_{F}^{o}$.

Proof. As in the proof of Theorem 5.8, let $\mathcal{A}$ be the ring of Witt vectors ${ }^{9}$ [Se 79 , II $\left.\S 6\right]$ with residue field an algebraic closure of the finite field $\mathbf{F}_{p}$. Using (4.4.2), we see - as in the proof of Theorem 5.8 - that it suffices to prove the Theorem after replacing $F$ by the residue field of $\mathcal{A}$ and

\footnotetext{
${ }^{9}$ The same remarks concerning the choice of $\mathcal{A}$ made in the footnote in Section 5.8 apply here as well; we could instead take for $\mathcal{A}$ any normal, local, Noetherian domain with infinite residue field of characteristic $p$ and field of fractions of characteristic 0 .
} 
$E$ by an algebraic closure of the field of fractions of $\mathcal{A}$, and after replacing $X_{F}$ and $X_{E}$ by nilpotent elements with the given Bala-Carter datum.

Again, let $G$ be a split reductive group scheme over $\mathcal{A}$ with the given root datum. Using Theorem 5.4 and the Bala-Carter Theorem, we may suppose that there is an equidimensional nilpotent section $X$ for which $X_{F}$ is conjugate to $X(s)$ and for which $X_{E}$ is geometrically conjugate to $X(\eta)$.

If $C=C_{G}(X)$ denotes the centralizer subgroup scheme, it now follows from Theorem 7.5 that $\left(C / C^{o}\right)_{\bar{s}} \simeq\left(C / C^{o}\right)_{\bar{\eta}}$. Thus, the component group of the centralizer in $G_{\bar{\eta}}$ of $X(\eta)$ is isomorphic to the component group of the centralizer in $G_{\bar{s}}$ of $X(s)$, as required.

\section{REFERENCES}

[Al 79] A. V. Alekseevskiü, Component groups of centralizers of unipotent elements in semisimple algebraic groups, Akad. Nauk Gruzin. SSR Trudy Tbiliss. Mat. Inst. Razmadze, 62 (1979). Collection of articles on algebra, 2.

[Bor 91] Armand Borel, Linear Algebraic Groups, 2nd ed., Grad. Texts in Math., vol. 126, Springer Verlag, 1991.

[Ca 93] Roger W. Carter, Finite groups of Lie type: conjugacy classes and complex characters, John Wiley \& Sons Ltd., Chichester, 1993. Reprint of the 1985 original.

[EGA IV] A. Grothendieck, Éléments de géométrie algébrique. IV. Étude locale des schémas et des morphismes de schémas. III, Inst. Hautes Études Sci. Publ. Math., 28 (1966), 255.

[SGA 1] A. Grothendieck, Revêtements étales et groupe fondamental (SGA 1), Documents Mathématiques 3, Société Mathématique de France, Paris, 2003. Séminaire de Géometrie Algébrique du Bois Marie. [Updated and annotated reprint of the 1971 original publication in Lecture Notes in Math., 224, Springer, Berlin.]

[SGA 3] A. Grothendieck and M. Demazure, Schémas en Groupes (SGA 3). I, II, III, Lectures Notes in Math., vol. 151, 152, 153, Springer Verlag, Heidelberg, 1965. Séminaire de Géometrie Algébrique du Bois Marie.

[Hu 95] James E. Humphreys, Conjugacy classes in semisimple algebraic groups, Math. Surveys and Monographs, vol. 43, Amer. Math. Soc., 1995.

[Ja 04] Jens Carsten Jantzen, Nilpotent orbits in representation theory, Lie Theory: Lie Algebras and Representations (J.-P. Anker and B. Orsted, eds.), Progress in Mathematics, vol. 228, Birkhäuser, Boston, 2004, pp. 1-211.

[Ja 03] Jens Carsten Jantzen, Representations of algebraic groups, 2nd ed., Mathematical Surveys and Monographs, vol. 107, American Mathematical Society, Providence, RI, 2003.

[Ke 78] George R. Kempf, Instability in invariant theory, Ann. of Math. (2), 108 (1978), no. 2, 299-316. 
[KMRT] Max-Albert Knus, Alexander Merkurjev, Markus Rost and Jean-Pierre Tignol, The book of involutions, Amer. Math. Soc. Colloq. Publ., vol. 44, Amer. Math. Soc., 1998.

[Li 02] Qing Liu, Algebraic geometry and arithmetic curves, Oxford Graduate Texts in Mathematics, vol. 6, Oxford University Press, 2002. Translated from the French by Reinie Erné.

[MS 03] George J. McNinch and Eric Sommers, Component groups of unipotent centralizers in good characteristic, J. Algebra, 260 (2003), no. 1, 323-337. Special issue celebrating the 80th birthday of Robert Steinberg. math.RT/0204275.

[MT 07] George J. McNinch and Donna M. Testerman, Completely reducible SL(2)homomorphisms, Trans. Amer. Math. Soc., 359 (2007), no. 9, 4489-4510.

[Mc 04] George J. McNinch, Nilpotent orbits over ground fields of good characteristic, Math. Annalen, 329 (2004), 49-85. arXiv:math. RT/0209151.

[Mc 05] Optimal SL(2)-homomorphisms, Comment. Math. Helv., 80 (2005), 391-426. arXiv:math. RT/0309385.

[Mc 06] On the centralizer of the sum of commuting nilpotent elements, J. Pure and Applied Algebra, 206 (2006), 123-140. arXiv:math.RT/0412283.

[Mil 80] James Milne, Étale Cohomology, Princeton University Press, 1980.

[Miz 80] Kenzo Mizuno, The conjugate classes of unipotent elements of the Chevalley groups $E_{7}$ and $E_{8}$, Tokyo J. Math., 3 (1980), no. 2, 391-461.

[Pr 03] Alexander Premet, Nilpotent orbits in good characteristic and the KempfRousseau theory, J. Algebra, 260 (2003), no. 1, 338-366. Special issue celebrating the 80th birthday of Robert Steinberg.

[Ri 82] R. W. Richardson, On orbits of algebraic groups and Lie groups, Bull. Austral. Math. Soc., 25 (1982), no. 1, 1-28.

[Se 79] Jean-Pierre Serre, Local Fields, Springer Verlag, 1979.

[Ser 05] Complète Réductibilité, Astérisque, 299 (2005), Exposés 924-937, pp. 195-217. Séminaire Bourbaki 2003/2004.

[So 98] Eric Sommers, A generalization of the Bala-Carter theorem for nilpotent orbits, Internat. Math. Res. Notices (1998), no. 11, 539-562.

[Sp 98] Tonny A. Springer, Linear algebraic groups, 2nd ed., Progr. in Math., vol. 9, Birkhäuser, Boston, 1998.

[St 68] Robert Steinberg, Endomorphisms of linear algebraic groups, Memoirs of the American Mathematical Society, No. 80, American Mathematical Society, Providence, R.I., 1968.

Department of Mathematics

Tufts University

503 Boston Avenue

Medford, MA 02155

USA

george.mcninch@tufts.edu, mcninchg@member.ams.org 